\title{
Studied X-FEM enrichment to handle material interfaces with higher order finite element
}

\author{
Kristell Dréau, Nicolas Chevaugeon, Nicolas Moës* \\ Institut de Recherche en Génie Civil et Mécanique, UMR CNRS 6183, Ecole Centrale de Nantes, B.P. 92101, 44321 Nantes Cedex3, France
}

\begin{abstract}
An approach to improve the geometrical representation of surfaces with the eXtended Finite Element Method is proposed. Surfaces are implicitly represented using the level set method. The finite element approximation is enriched by additional functions through the notion of partition of unity, to track material interfaces. Optimal rate of convergence is achieved with curved geometries, using linear elements and linear level set in elements. In order to accelerate the convergence, the order of approximation shape functions is increased, while keeping the same computational mesh. The level set is represented on a finer submesh than the finite element mesh. A special attention to integration procedure is necessary. A new enrichment function is introduced to represent the behavior of curved material interfaces. Numerical examples including free surfaces and material interfaces in 2-D linear elasticity are presented to study convergence rates.
\end{abstract}

Keywords: eXtended Finite Element Method Level set ,High order approximation, Free surface, Material interface, Curved geometries

\section{Introduction}

Mesh generation of complex geometries can be very timeconsuming, within a classical finite element analysis. The main difficulty arises from the necessity of the mesh to conform to physical surfaces. Discontinuities such as holes, cracks and material interfaces may not cross mesh elements. Moreover, local refinements close to discontinuities and mesh modification to track the geometrical and topological changes in crack propagation problems for example, can be difficult. Also, when geometries evolve and history dependent models are used, robust methods to transfer the solution to the new mesh are needed. This issue is particularly significant, since computed fields defined on these discontinuities are often the most important ones.

In order to improve the geometrical representation of surfaces, and particularly with complex geometries including curves, a solution is to locally refine the mesh, close to discontinuities, i.e. to decrease element size. Several methods of adaptive h-refinements were developed (for example $[9,25,30]$ ), by subdividing elements or remeshing. The position of nodes can also be adjusted in order to improve the geometrical representation while keeping their number constant; this is called r-refinement.

Another mean to reduce geometrical errors is to introduce curved elements in the mesh. Elements with curved boundaries are used to

\footnotetext{
* Corresponding author.

E-mail addresses: kristell.dreau@ec-nantes.fr (K. Dréau), nicolas.chevaugeon@ec-nantes.fr (N. Chevaugeon), nicolas.moes@ec-nantes.fr (N. Moës).
}

represent curved geometries, increasing the degree $p$ of geometrical shape functions and the number of geometrical nodes on boundaries. This technique allows the use of meshes with fewer elements. Adaptive $p$-refinement, also called p-method, is presented in [30]. Usually, these shapes functions are constructed with Legendre polynomials. With iso-parametric element, shape functions are the same for the field approximation and the geometry. Curvilinear mesh generation encounters two main issues $[4,26]$ : the respect of the geometrical representation, and the generation of a valid mesh. For example, gaps and overlaps have to be removed from meshes. Integration of functions on these elements must be computed with caution $[14,30]$. If an order $p$ increases, oscillations may nevertheless appear.

Recent studies about $p$-version mesh generation develop the use of Bezier polynomials to represent curves and surfaces [17,18]. Increasing the geometric approximation order and subdividing elements become easier, due to properties of Bezier polynomials. New mapping procedures have to be defined. However, mesh generation and geometrical approximation still have to be controlled.

Isogeometric analysis [11] gets rid of Lagrange polynomials drawbacks: geometry is exactly represented by NURBS and refinements simplified. However, this geometrical benefit leads to additional difficulties in numerical integration [12].

Several numerical methods were developed, in order to get rid of mesh difficulties. An idea was, for example, to base approximations on a set of nodes without creating any mesh. These kinds of methods are called Meshfree Methods [8]. Therefore, hp-adaptivity becomes easier because of the absence of a mesh [5]. However, difficulties arise from numerical integration and treatment of essential boundary conditions. 
For sake of mesh simplifications, the eXtended Finite Element Method (X-FEM) was developed 10 years ago, initially for crack propagation $[1,3,22]$.

Within the X-FEM, surfaces that are not represented explicitly by mesh boundaries can be implicitly represented by the iso-zero values of a level set function. This is particularly useful for moving interfaces such as crack surfaces in crack propagation analysis [29]. The finite element approximation is enriched by additional functions through the notion of partition of unity [20] to represent, for example, discontinuities at interfaces [28] or asymptotic expansions terms near crack tip [29] to improve convergence rate. X-FEM is a good alternative to the classical finite element method for solving mechanical problems with complex or evolving geometries.

Enrichment functions are usually defined with the help of the level set functions to access the distance to the interface at any given point. Although interfaces do not have to be meshed, the correct integration of the element stiffness matrix, for elements enriched by a discontinuous function along an interface, needs to be done carefully. These elements are split for integration purpose along the iso-zero level set, creating a set of integration cells called partition. A regular integration is done separately on each cells of the partition.

In the case of free surfaces [28], the level set is only used to construct the partition and to determine if integration cells of this partition are in the material or in the hole. On the contrary, material interfaces and cracks need additional functions for displacement field $[3,21]$ depending on level set values on element nodes.

Difficulties to represent the surface in the classical finite element method are, in the context of X-FEM, partly shifted on the integration procedure. A method for eliminating the subdivision of elements during the integration of the stiffness matrix is presented in [32]: discontinuous and non-differentiable functions are replaced with equivalent polynomials (exact equivalence for triangular and tetrahedral elements). However, it applies only for linear shape functions and a straight discontinuity across elements. Nevertheless, X-FEM gives good results with linear elements and linear level set in elements: optimal rate of convergence is achieved with curved geometries [28].

In order to accelerate the energy error convergence, $p$-versions of X-FEM were developed. In $[13,15,27]$ for example, high order X-FEM for straight cracks also reaches an optimal rate of convergence. If the crack has not a flat surface, the error is reduced with shape functions of higher order without improving the geometrical representation, but the optimal rate of convergence is not achieved. The problem is of course related to the well known fact that, in the framework of classical finite element method, when increasing the order of the approximation field, the order of representation of the geometry must be increased accordingly to get optimal rate of convergence. Isoparametric elements have an energy error norm on regular problems with curved boundary that converges as $O\left(h^{p}\right)$, where $h$ is the element size and $p$ the polynomial basis order (see [33]).

To get optimal rate of convergence when curved interfaces are represented with level set, the obvious solutions would be to increase the order of the level set representation near the iso-zero values of the level set, as in [31]. In this case, the integration problem can become quite difficult: within an element, the iso-zero surface can have complex shape and topology. In [16], X-FEM is developed with spectral elements, to deal with arbitrary discontinuities: optimal rate of convergence in energy norm are not reached for curved discontinuities, and integration is more delicate. The influence of the geometry description within the Finite Cell Method is investigated in [6] for three-dimensional problems with curved boundaries: using an implicit representation and using a voxel model that is promising for biomechanics simulations.

$\mathrm{X}$-FEM is extended to high order approximations in [2] for arbitrary curved strong and weak discontinuities. Elements crossed by a discontinuity are divided into subcells with one curved side. Optimal convergence is reached in the first case and a close-tooptimal one in the second case using the Corrected X-FEM [7].
The alternative approach proposed here consists in representing the level set on a finer sub-mesh than the finite element mesh, while keeping the level set representation piecewise linear by element (without adding any degree of freedom to the mechanical field). This is used in [19] to localize an interface. This idea was also developed in hp Generalized FEM [24]: representation of crack surfaces is controlled by a Delaunay tetrahedralization of elements cut by the discontinuity.

$\mathrm{X}$-FEM is presented in the next section, with a brief recall of linear elasticity. A new strategy is then introduced to use high order X-FEM and applied to domains with curved boundaries. Numerical applications of free surfaces and material interfaces are developed within the framework of linear elasticity with polynomial approximations up to order three.

\section{Extended finite element method}

\subsection{Model problem}

The formulation is written within the framework of linear elastostatic. Consider a domain $\Omega$, with boundary $\Gamma$, representing a linear elastic, homogeneous and isotropic solid. The domain is submitted to impose displacement $\bar{u}$ on $\Gamma_{u}$ and imposed tractions $\bar{t}$ on $\Gamma_{t}$. We study the case without volumic force, in small strain. Stresses, strains and displacements are respectively denoted by $\sigma, \varepsilon$ and $u$.

The problem is to find the admissible displacement field which satisfies the following equations:

- Equilibrium equations:

$\nabla \cdot \sigma=0$ on $\Omega$

$\sigma \cdot n=\bar{t}$ on $\Gamma_{t}$,with $n$ the external normal

- Kinematics equations:

$$
\begin{aligned}
& \varepsilon(u)=\frac{1}{2}\left(\nabla u+(\nabla u)^{T}\right) \text { on } \Omega \\
& u=\bar{u} \text { on } \Gamma_{u}
\end{aligned}
$$

- Constitutive law:

$\sigma=C: \varepsilon$, on $\Omega$, where $C$ is Hooke's tensor

Let $\mathcal{U}$ be the space of admissible displacement field, and $\mathcal{U}_{0}$ the space of kinematically admissible test functions, vanishing on $\Gamma_{u}$. The weak form of equilibrium elastostatic and boundary conditions can be written as:

Find $u \in \mathcal{U}$ such that

$\int_{\Omega} \varepsilon(u): C: \varepsilon(v) d \Omega=\int_{\Gamma_{t}} \bar{t} \cdot v d \Omega, \quad \forall v \in \mathcal{U}_{0}$

\section{2. eXtended Finite Element Method}

Within the finite element method, the domain $\Omega$ is divided into elements, called finite elements and constituting a mesh. The displacement field is approximated on these elements:

$u(x)=\sum_{i}^{n} \phi_{i}(x) u_{i}$

where $\phi_{i}(x)$ are the finite element shape functions, and $u_{i}$ the nodal displacements.

Within X-FEM, the mesh does not necessarily conform to internal discontinuities (such as holes, cracks, and material interfaces), which allows the use of simple meshes (structured or not). Moreover, the finite element approximation is enriched by additional functions 
(through the partition of unity [20]) that model the behavior of boundaries, as follows:

$u^{h}(x)=\sum_{i \in N} \phi_{i}(x) u_{i}+\sum_{j \in N^{g}} \phi_{j}(x) F(x) a_{j}$

where $F(x)$ is the enrichment function; $a_{j}$ are additional degrees of freedom, for enriched nodes; $N$ represents the set of the nodes of the mesh, and $N_{g}$ the set of enriched nodes.

For example, in the case of material interfaces, only nodes belonging to elements crossed by the interface are enriched. If the mesh conforms to discontinuities, there is no enrichment on nodes, and classical finite element method is recovered.

As surfaces are not represented explicitly by mesh boundaries, a function, called level set $\varphi(x, t)$ [23], is introduced to track them. This function usually gives the signed distance to the interface at any point of the domain (Fig. 1). Consequently, the interface location is given by the iso-zero value of the level set: $\varphi(x, t)=0$.

\section{High order X-FEM with complex geometries}

\subsection{A new approach}

For the moment, X-FEM is mostly used with linear approximation shape functions $\phi_{i}(x)$ and linear level set. Optimal (or nearly optimal) rates of convergence are reached in the case of holes, material interfaces and cracks $[15,21,28,29]$ for straight cracks).

Our purpose is to accelerate the convergence of X-FEM, using high order shape functions for the finite element approximation. However, the optimal rate of convergence can't be reached without improving the geometrical representation, geometrical errors becoming more important than approximation errors.

Instead of using an exact representation of the domain (as [11] for example), the finite element method utilizes a polynomial representation of the geometry. The first idea for the representation of complex geometries with X-FEM would be to use elements with curved boundaries.

These elements can be easily generated by adding geometrical nodes on edges, with hierarchical shape functions. $p$-refinement does not need to be uniform throughout the domain. Energy error norm on regular problems converges as $O\left(h^{p}\right)$ with iso-parametric elements, where $h$ is the element size and $p$ the polynomial basis order. However, the mesh generation might become quite difficult with this kind of elements. Indeed, Fig. 2 illustrates a classical problem of geometrical representation: when the edge 1 is improved, overlap can appear.

In reference [31], level set of order two are used to model interfaces, providing a more accurate representation. In [27], higher order elements are applied to curved cracks problems (six-node triangular elements) with quadratic shape functions. Although the energy error is reduced, the rate of convergence is not improved. Furthermore, it is shown that the use of linear shape functions for the enrichment function gives better results, even though higher order shape functions are used for the continuous approximation.

Instead of using a level set representation of higher order, leading to complex shapes of iso-zero surfaces and integration difficulties, a strategy is presented to get closer to the real geometry of the

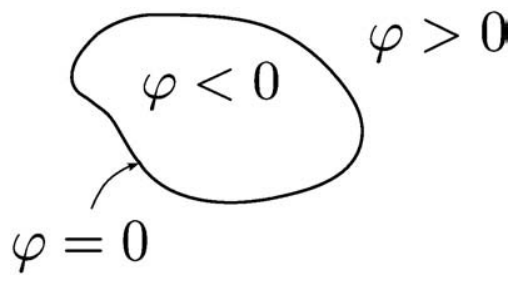

Fig. 1. Level set $\varphi(x)$. discontinuity while keeping a linear representation of the geometry. This consists in representing the level set on a finer mesh than the finite element mesh, without additional degree of freedom for the mechanical field. Thus, representation of discontinuities such as holes boundaries, material interfaces or cracks is improved in a simple way.

\subsection{Geometrical representation using level sets}

The level set is interpolated on elements with finite element shape functions. These geometrical shape functions are generally the same than approximation shape functions (case of iso-parametric elements). For now, within X-FEM, the level set is linear on elements (linear shape functions). Therefore, the mesh has to be fine enough to accurately locate the interface.

The aim is to improve the geometrical representation while keeping the same computational mesh, and therefore without adding any degree of freedom. The level set is computed on a finer mesh than the computational mesh, called "geometrical grid", and its elements are called "cells". Initially, the geometrical grid is a copy of the computational mesh. If the geometrical representation has to be improved, the geometrical grid has to be refined. Thus, each cell crossed by the iso-zero is split into cells of same nature. For example, a triangle in 2D is divided in 4 triangles (quadtree refinement). Level set values are then computed on the new nodes. The procedure is done recursively up to a user defined maximum depth, forming the final geometrical grid (see Fig. 5). Each element of the analysis is then linked to an octree-like partition where the level set is represented and on which the integration procedure is applied for each sub-element. The approximation of the iso-zero surface is then improved at each level of refinement of the geometrical grid. The interface mesh representing the boundary of a hole is plot on Fig. 3, with no refinement, and a level of refinement equal to 1 and 2 . Refining the geometrical grid improves the representation of the interface while keeping a level set piecewise linear on elements.

\subsection{Numerical integration}

Numerical integration has to be handled with care in X-FEM. Indeed, discontinuous functions can't be directly integrated on elements. Therefore, cells of the geometrical grid cut by the iso-zero value of the level set are split in integration cells (Fig. 4), creating partitions tied to mesh elements, as presented in [22]. A regular integration is thus done in these integration cells (Gauss integration), on both sides of the discontinuity. For example, an element crossed by the boundary of a hole is split in integration cells, and integration is performed only on the cell belonging to the material (i.e. not in the void).

If the geometrical grid is refined according to the previous description, integration operations are applied to cells: integration cells are created, and gathered in partitions linked to mesh elements. This process is explained in Fig. 5.

Finally, partition cells are classified depending on their distances to the interface. For example, in the case of holes, partition cells are classified in two groups: cells in the material or in a hole. Thus, integration is performed only on partition cells in the material, those in holes being ignored.

Next sections prove the validity of this high order approach. The study of benchmark elasticity problems allows one to verify the convergence of energy norm with high order X-FEM. First, the case of curved free surfaces is observed with an infinite plate with a circular hole under uniaxial tension. The case of curved material interfaces presents a more sensitive issue and is afterward studied with circular inclusion. All results are presented using GMSH [10] as a post-processing tool.

\section{Free surfaces}

First, the treatment of free surfaces with X-FEM is considered. In order to check the validity of this proposed method, we are interested 

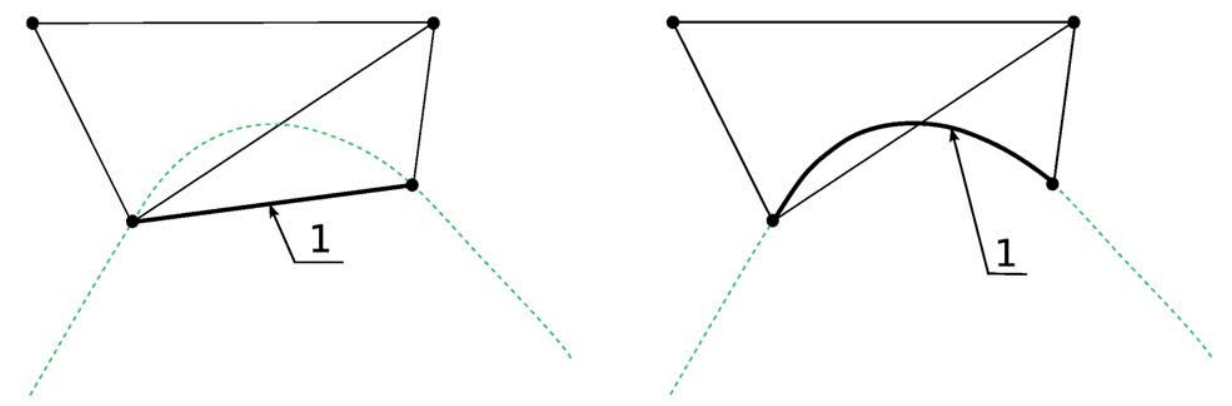

Fig. 2. Boundary of a hole with finite elements.
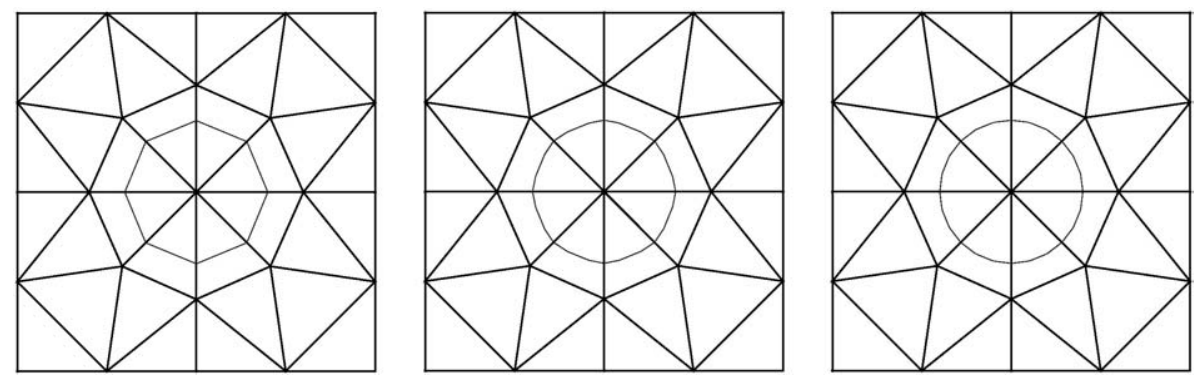

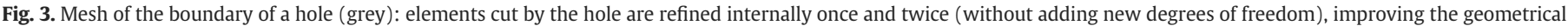
representation of the hole.
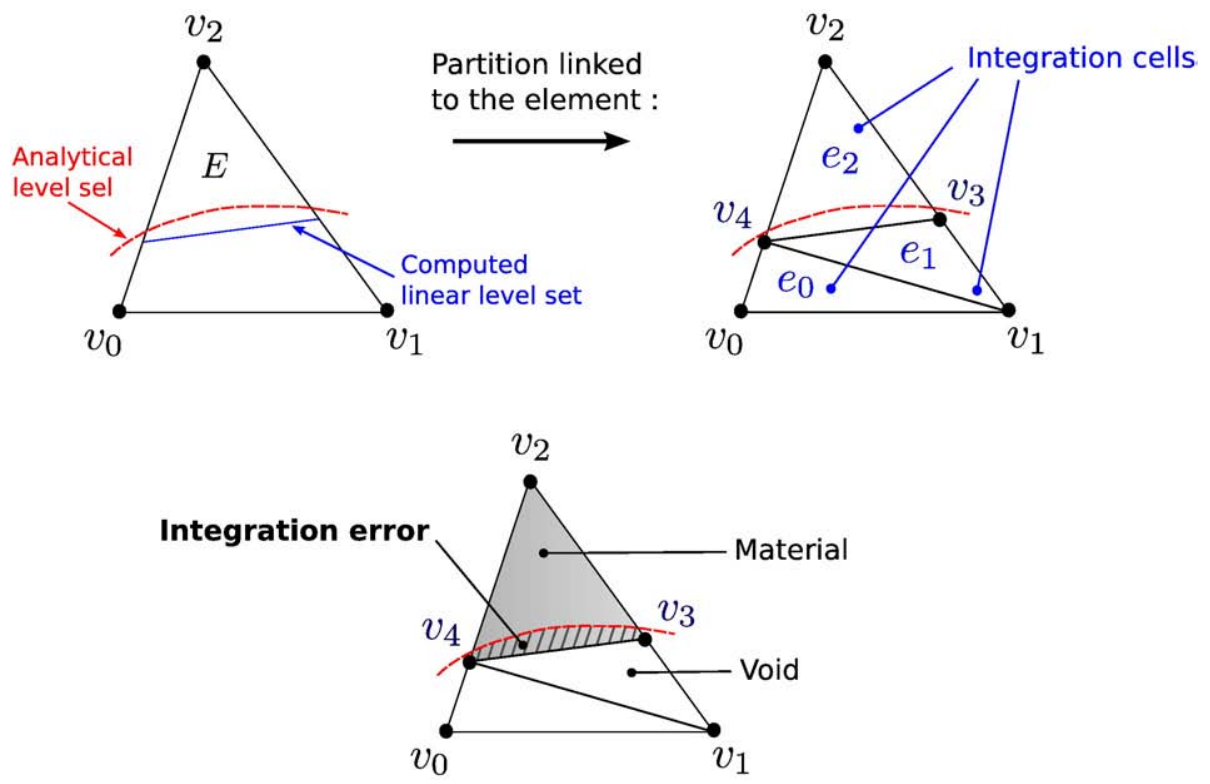

Fig. 4. Numerical integration with X-FEM: creation of a partition.

in studying the convergence of the solution for an infinite plate with a hole under uniaxial tension. Thus, the classical finite element method and the extended finite element method can easily be compared, since an analytical solution exists.

The case of free surfaces can be easily handled with X-FEM $[3,28]$. Nodes for which the support is totally inside voids are wiped out of the computation.

\subsection{Infinite plate with a hole under uniaxial tension}

We consider an infinite plate with a circular hole under uniaxial tension $\sigma_{\infty}=1$ along axis $\mathrm{x}$ (see Fig. 6). This plate is modeled by a square domain of side $L$ with a circular hole of radius $a$ in its center. Exact tractions are imposed on boundaries of the domain.

Exact solution is shown in [30]. Components of exact stress tensor are:

$\sigma_{x x}(r, \theta)=1-\frac{a^{2}}{r^{2}}\left(\frac{3}{2}(\cos (2 \theta)+\cos (4 \theta))\right)+\frac{3}{2} \frac{a^{4}}{r^{4}} \cos (4 \theta)$

$\sigma_{y y}(r, \theta)=-\frac{a^{2}}{r^{2}}\left(\frac{1}{2}(\cos (2 \theta)-\cos (4 \theta))\right)-\frac{3}{2} \frac{a^{4}}{r^{4}} \cos (4 \theta)$ 
Computational mesh :

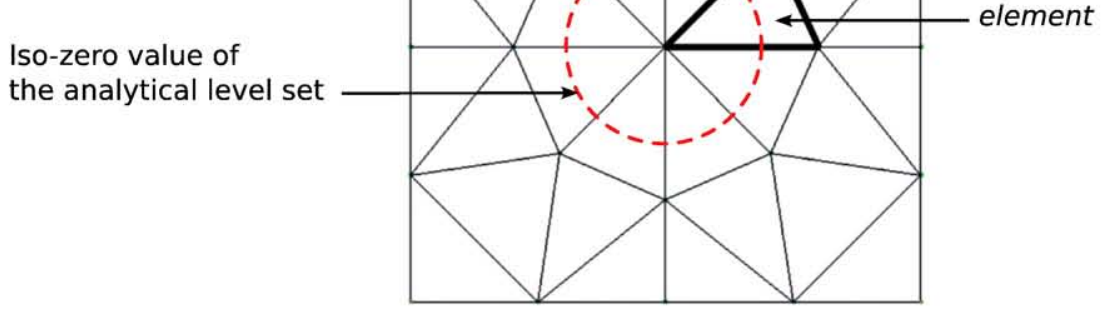

Recursive refinements :

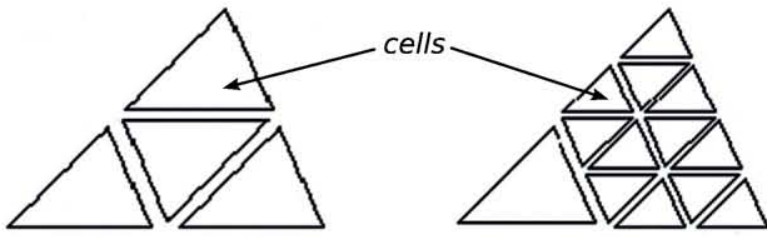

First refinement

Second refinement

Creation of a partition tied to the mesh element :

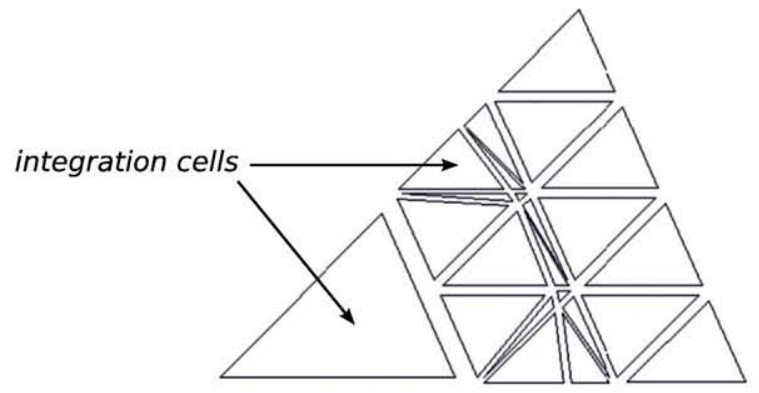

Classification of integration cells :

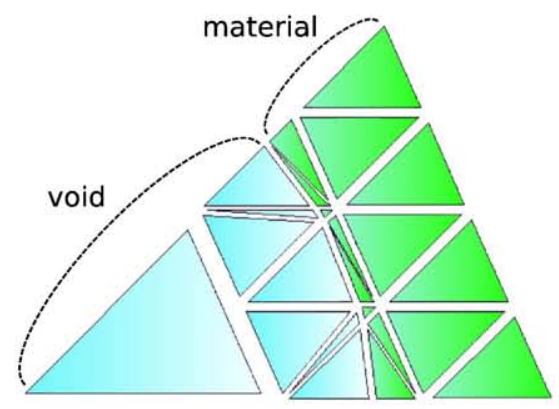

Fig. 5. Numerical integration with high order X-FEM: creation of a partition.

$\sigma_{x y}(r, \theta)=-\frac{a^{2}}{r^{2}}\left(\frac{1}{2}(\sin (2 \theta)+\sin (4 \theta))\right)+\frac{3}{2} \frac{a^{4}}{r^{4}} \sin (4 \theta)$

And exact displacements read:

$u_{x}(r, \theta)=\frac{a}{8 \mu}\left[\frac{r}{a}(\kappa+1) \cos (\theta)+2 \frac{a}{r}(1+\kappa) \cos (\theta)+\cos (3 \theta)-2 \frac{a^{3}}{r^{3}} \cos (3 \theta)\right](5)$
(4) $\quad u_{y}(r, \theta)=\frac{a}{8 \mu}\left[\frac{r}{a}(\kappa-3) \sin (\theta)+2 \frac{a}{r}(1-\kappa) \sin (\theta)+\sin (3 \theta)-2 \frac{a^{3}}{r^{3}} \sin (3 \theta)\right]$

with:

- $a=0.4$

- $E=10^{5}$ : Young Modulus

- $\nu=0.3$ : Poisson's ratio 


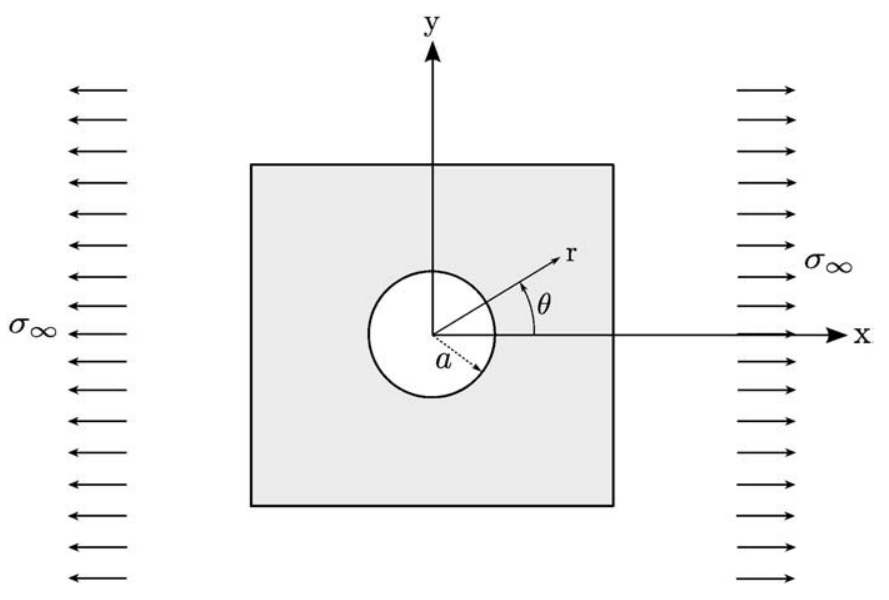

Fig. 6. Infinite plate with a hole under uniaxial tension.

- $\mu$ : shear modulus

- $\kappa$ : Kolosov constant $\kappa=3-4 \nu$ (simulations are carried out in plane strain)

\section{Results}

In order to compare precision of the finite element solution with the one computed with X-FEM, convergence of the relative error in the energy norm is studied, for square regular meshes with $8,16,32$, 64 and 128 elements (triangular elements) by side. The side length is 2. Numerical experiments are done with polynomial approximation up to order three. Energy error norm is given by:

$e=\frac{\sqrt{\int_{\Omega} \varepsilon\left(u^{h}-u^{e x}\right): C: \varepsilon\left(u^{h}-u^{e x}\right) d \Omega}}{\sqrt{\int_{\Omega} \varepsilon\left(u^{e x}\right): C: \varepsilon\left(u^{e x}\right) d \Omega}}$

Fig. 7 presents the relative energy error functions of the mesh element size, convergence rates being computed from errors of the two finest meshes (with 64 and 128 elements). Using high order finite element approximation without improving the geometrical representation does not give good results, in term of convergence rate of the energy error norm. The use of approximation functions of orders 2

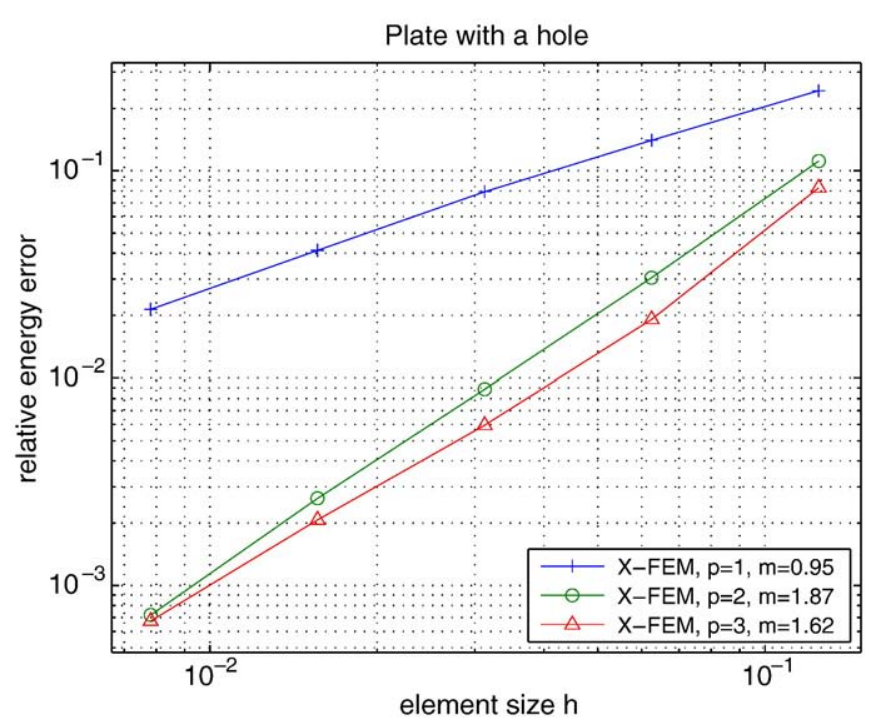

Fig. 7. Energy error norm for a plate with a hole, with orders of approximation 1, 2 and 3. and 3 improves the convergence rate compared with an approximation of order 1. However, it does not reach the classical finite element rate (Fig. 7), that is $O\left(h^{p}\right)$. We note that the quadratic approximation reaches a convergence rate quite close to the finite element one: average rate of 1.87 . On the contrary, the cubic approximation converges with a rate of 1.62 . Note that these rates correspond to a particularly penalizing case. Indeed, using a circular inclusion radius of 0.4 , the iso-zero value of the level set passes over some elements nodes, which is slightly penalizing.

Using the present method, improving the geometrical representation of the boundary of the hole reduces geometrical errors and consequently global error. Fig. 8 presents the convergence of the relative energy error functions of the mesh element size, with cubic approximation shape functions, for different level of precision of the geometrical representation (recursive refinement of the geometrical grid). The more refined is the geometrical grid, the smaller is the relative error norm. Choosing an optimal refinement level, depending on the order of approximation $p$ and on the element size $h$, allows to accelerate the convergence of energy error functions of the mesh element size (see Fig. 9): energy error converges with a quasi-optimal rate of 2.93 .

We note that the condition number could be deteriorated if elements or part of them are located within the hole. This is why elements completely in the hole are removed from the computation, and integration is performed only in partition cells in the material. Moreover, a loop is done on mesh edges. If the intersection point between an edge and the iso-zero value of the level set is very close to one of the two nodes (distance smaller than $10^{-3} h$, with $h$ the edge size), the level set value of the node is modified and equal to zero. This node is "moved" on the interface. Then, precision of computations is assured by a direct solver. Finally, condition number is not deteriorated.

Thus, we show that optimal convergence rate can be achieved with an optimal geometrical representation of the boundary of the hole.

Global error is made up of interpolation error and geometrical error. Approximation error decreases as $\left(h_{a}\right)^{p}, h_{a}$ being the relative approximation element size, and $p$ the degree of approximation shape functions. Numerical experiments show that geometrical error decreases as $\left(h_{g}\right)^{2}, h_{g}$ being the relative geometrical cell size. With a linear approximation and a linear geometry on X-FEM elements (and also for iso-parametric elements), relative approximation and geometrical elements are the same: $h_{a}=h_{\mathrm{g}}$. This relation is no longer correct with higher order approximation. A method to find the correct

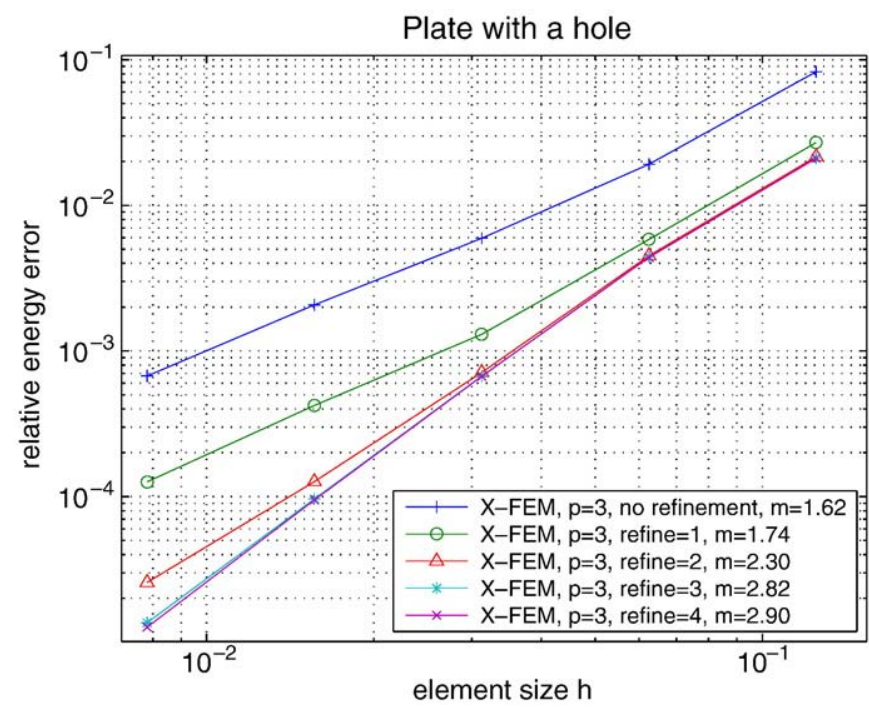

Fig. 8. Energy error norm for a plate with a hole using cubic approximation shape functions and different refinement level of the geometrical grid. 


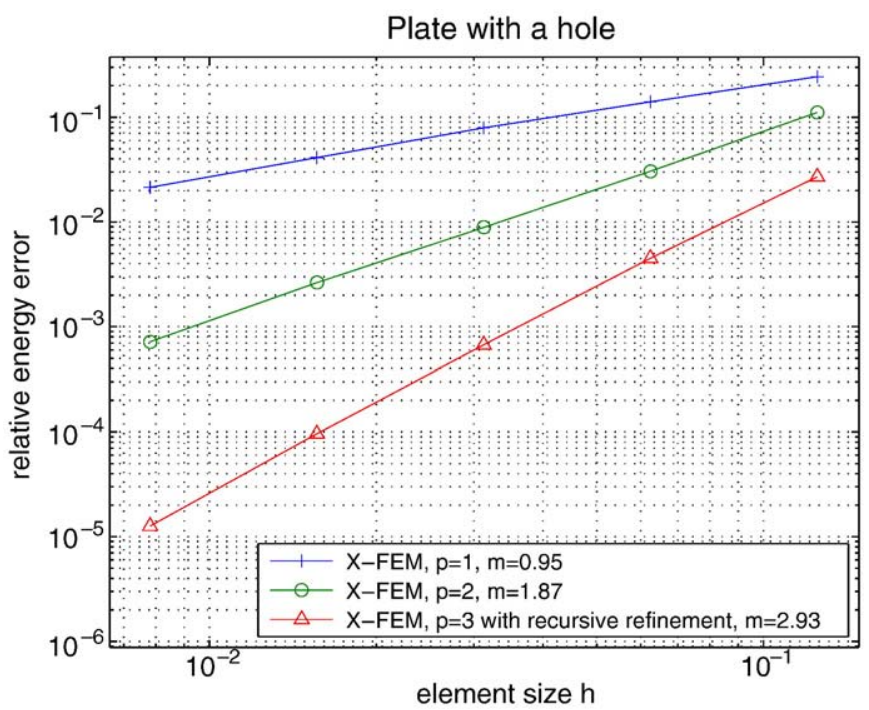

Fig. 9. Energy error norm for a plate with a hole using orders of approximation 1, 2 and 3 , improving the geometrical representation.

geometrical cell size, that is to say the correct refinement level, is presented.

Consider a mesh of relative element size $h_{a}$. In order to find the geometrical cell size, let us consider geometrical and approximation errors orders to be equal:

$h_{g}^{2}=h_{a}^{p}$

Therefore, the relative geometrical cell size is:

$h_{g}=\left(h_{a}\right)^{\frac{p}{2}}=\left(\frac{1}{n}\right)^{\frac{p}{2}}=h_{a}\left(\frac{1}{n}\right)^{\frac{p}{2}-1}$

For example, considering quadratic shape functions $(p=2)$, we have $h_{g}=h_{a}$. Indeed, the relative energy error converges with the optimal rate, without the need to improve the geometry.

With cubic shape functions $(p=3)$, the relation becomes:

$h_{g}=\left(\frac{1}{n}\right)^{\frac{1}{2}} h_{a}$
Fig. 10 shows theoretical division of the approximation element and the chosen refinement level for each mesh, as element size can only be divided by $2^{r}$ during geometrical refinement. The smaller is the mesh element size, the bigger is the refinement level. For example, the geometrical grid is refined twice using cubic shape functions and a square mesh with 32 elements by side.

This relation just provides $h_{g}=h_{g}\left(h_{a}\right)$, to maintain the optimal convergence rate. However, it does not depend on the curvature of the interface. A straight interface does not need refinement, as geometry is correctly represented with linear level set. On the contrary, a highly curved interface has to be well represented on elements thanks to a fine geometrical grid. A reflexion has to be lead on a correct rule to define the refinement level depending on local curvature of interface.

Thus, dealing with free surfaces, the proposed method allows one to use higher order approximation shape functions while keeping the optimal convergence rate of classical finite element methods while avoiding the generation of curved meshes.

\section{Material interfaces}

\subsection{X-FEM for material interface}

The X-FEM approximation requires an enrichment function, able to represent the behavior of an interface between two materials. Indeed, as the mesh does not conform to the interface, some elements overlap the two materials. An enrichment term is added to the finite element approximation on nodes of elements cut by the interface, introducing additional degrees of freedom $a_{j}$, and an enrichment function $F(x)$.

$u^{h}(x)=\sum_{i \in N} \phi_{i}(x) u_{i}+\sum_{j \in N^{g}} \phi_{j}(x) F(x) a_{j}$

where $u_{i}$ are the degrees of freedom and $\phi_{i}(x)$ the approximation shape functions.

Elements cut by the discontinuity have to be split into sub-elements belonging to one or the other material, for numerical integration.

Displacement is continuous across a material interface, with a discontinuous gradient. In the case of material interface, the level set $\varphi(x)$ is not only used to track the geometry of the interface, but it is also used to build enrichment functions. In [21], an enrichment strategy is proposed to model discontinuities along material interfaces. The enrichment function (10) was tested for a 2D circular and a $3 \mathrm{D}$ spherical inclusion under tension in an infinite domain. This function is continuous, with a discontinuous derivative across the interface (Fig. 11). It is maximal on the interface, and equal to zero on

\begin{tabular}{c|c|c|c}
$\begin{array}{c}n \text { elements by side } \\
\text { of the mesh }\end{array}$ & $\begin{array}{c}\text { approximation } \\
\text { element size } h_{a}\end{array}$ & $\begin{array}{c}\text { geometrical } \\
\text { element size } h_{g}\end{array}$ & refinement level \\
\hline 8 & $\frac{1}{8}$ & $\frac{h_{a}}{2: 8}$ & 1 \\
\hline 16 & $\frac{1}{16}$ & $\frac{h_{a}}{4}$ & 2 \\
\hline 32 & $\frac{1}{32}$ & $\frac{h_{a}}{5: 6}$ & 3 \\
\hline 64 & $\frac{1}{64}$ & $\frac{h_{a}}{8}$ & 3 \\
\hline 128 & $\frac{1}{128}$ & $\frac{h_{a}}{11: 3}$ & 4
\end{tabular}

Fig. 10. Free surfaces: refinement level for a cubic approximation. 


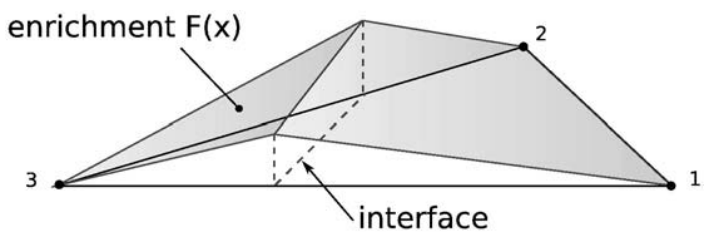

Fig. 11. Initial enrichment.

element nodes, edges and faces not crossed by this interface. The convergence rate of energy error is observed as close-to-optimal one.

$F(x)=\sum_{i}\left|\varphi_{i}\right| \phi_{i}(x)-\left|\sum_{i} \varphi_{i} \phi_{i}(x)\right|$

with $\varphi_{i}$ the level set value on element nodes.

\subsection{High order X-FEM for straight interfaces}

Before studying curved material interfaces, we are interested in using high order approximation shape functions simply with straight material interfaces. In this case, the transition to high order X-FEM is obvious. Indeed, as geometry is represented by a linear function, the enrichment function has to remain the same when the geometrical grid is refined. Thus, the previous enrichment term [21] is still convenient.

For example, consider a plate of side $L=2$, and section $S$ with two materials 1 and 2: $E_{1}=1, E_{2}=10, \nu_{1}=\nu_{2}=0$. Plain-strain condition is assumed. It is embedded on the left side and a variable volumic load is imposed on the plate (Fig. 12): $\|\vec{f}\|=f\left(\frac{x}{L}\right)^{2}$.

Exact displacements in the two materials are:

$$
\begin{aligned}
& u_{1}(x)=\frac{f}{3 E_{1} S L^{2}}\left(L^{3} x-\frac{x^{4}}{4}\right) x \\
& u_{2}(x)=\frac{f}{3 E_{2} S L^{2}}\left(L^{3} x-\frac{x^{4}}{4}\right)+\frac{31 f L^{2}}{192 S}\left(\frac{1}{E_{1}}-\frac{1}{E_{2}}\right) x
\end{aligned}
$$

Computations are done on squared irregular meshes, with 5,10 , 20,40 , and 80 elements (triangular elements) by side $(L=2)$, with linear, quadratic and cubic polynomial approximations. Element edges of theses meshes do not align with the straight interface, as shown in Fig. 13: iso-zero value of the level set is plot on a mesh with 10 elements by side. Results are presented in Fig. 14: energy norm converges with close-to-optimal rates, when higher order approximations are used.

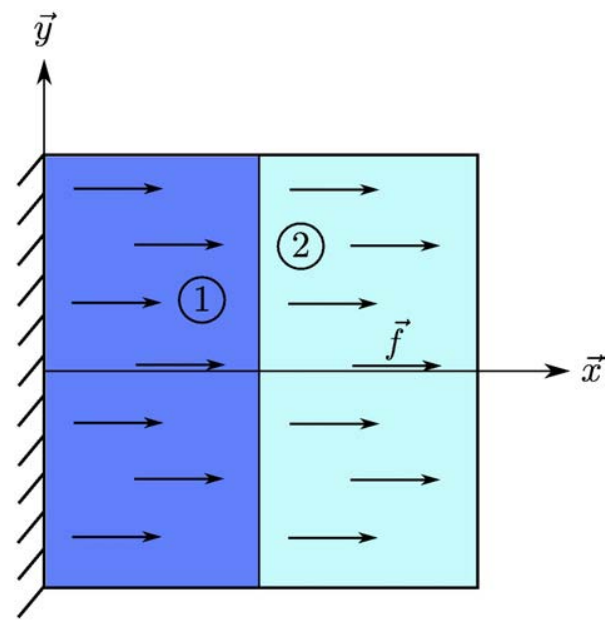

Fig. 12. Plate with a material interface and a variable volumic load.

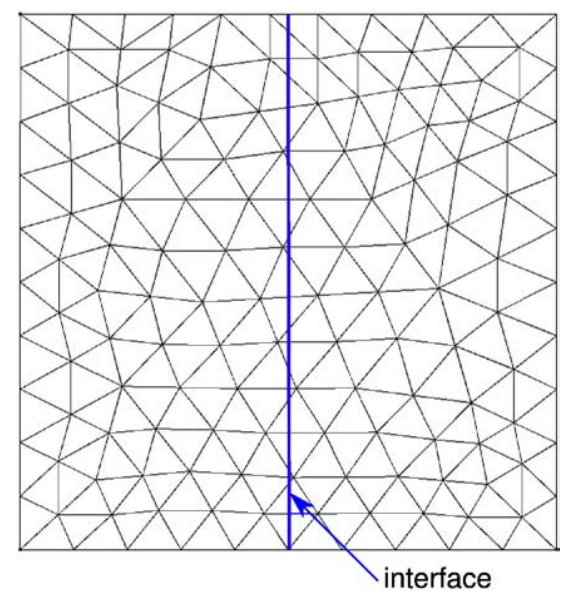

Fig. 13. Iso-zero value of the level set.

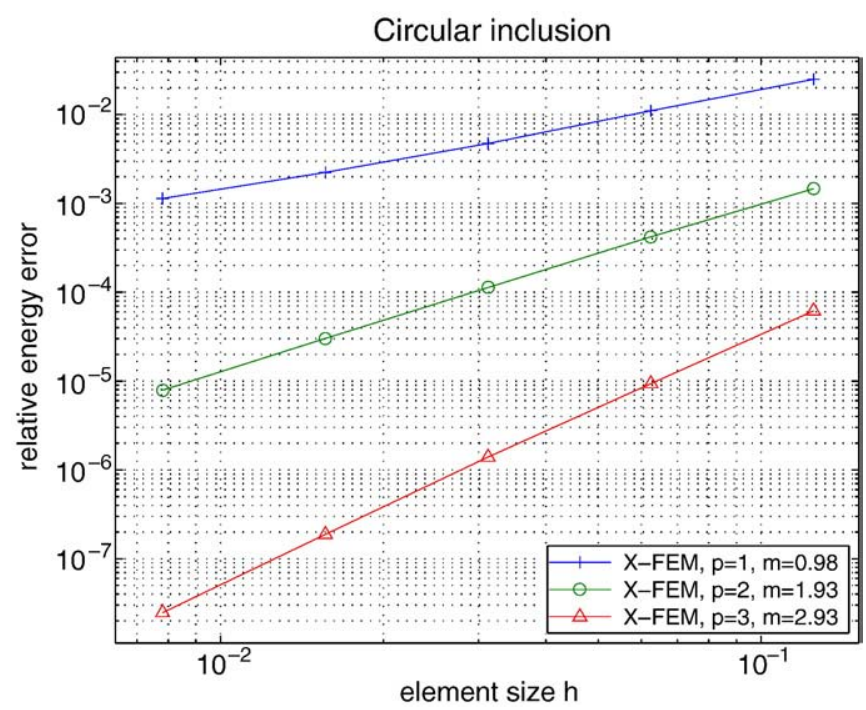

Fig. 14. Energy error norm for the plate with a straight material interface.

\subsection{New enrichment strategy for curved material interfaces}

Enrichment described in Eq. (10) is used with a linear representation of the geometry. In order to use high order approximation functions, the same strategy than free surfaces is applied for curved material interfaces. That is to say that the geometrical grid is locally refined while keeping a piecewise linear level set representation by element. The level set is computed on the geometrical grid, so that its representation is more precise than if it is computed on the original mesh (without refinement). However, the previous enrichment function is not suitable anymore because of a gap between the position of the iso-zero value of the level set and the discontinuity on the element (Fig. 15).

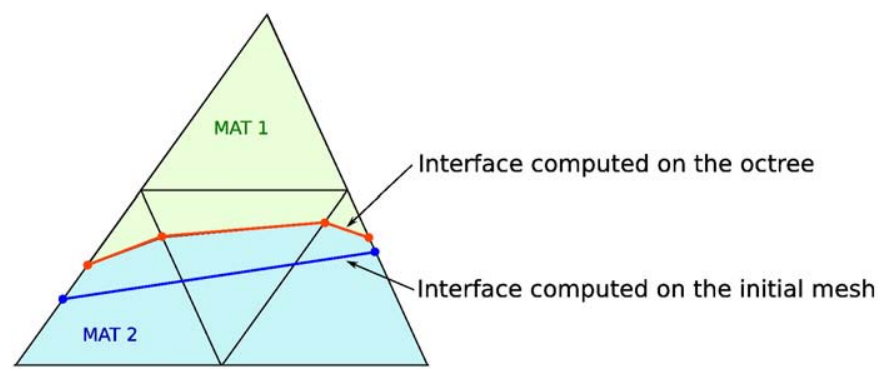

Fig. 15. Iso-zero value of the level set computed on the initial mesh and the octree (refined once) 
A new enrichment strategy is developed to solve this problem and to obtain optimal convergence.

Elements that are not crossed by the iso-zero value of the level set are not enriched. As the enrichment function is continuous over the domain, it has to be equal to zero on nodes, edges and faces, whose adjacent elements are not enriched. The derivative is discontinuous across the interface, whose position is given by the iso-zero value of the level set. Moreover, it has to be valid whatever the refinement level of the geometrical grid. This new enrichment is here proposed for triangle elements in 2D. It will be presented for tetrahedron elements in 3D in a forthcoming paper.

Different configurations are considered, functions of the position of the iso-zero value of the level set in relation to the element.
To build such function, we start by something similar to the enrichment in the linear case: the enrichment term depends on both level set nodal values and its values on the geometrical grid.

$$
\hat{F}(x)=\sum_{i \in \text { mesh }}\left|\varphi_{i}\right| \phi_{i}(x)-\left|\sum_{j \in \text { octree }} \varphi_{j} \phi_{j}(x)\right|
$$

This function (11) has a discontinuous derivative across the iso-zero value of the level set, and is equal to zero on mesh element nodes. It is different to zero on edges, and particularly on edges that are not cut by the iso-zero value of the level set. However, mesh elements that are not crossed by the interface are not enriched. Therefore, enrichment function on edges that are not cut has to be equal to zero. Theses edges

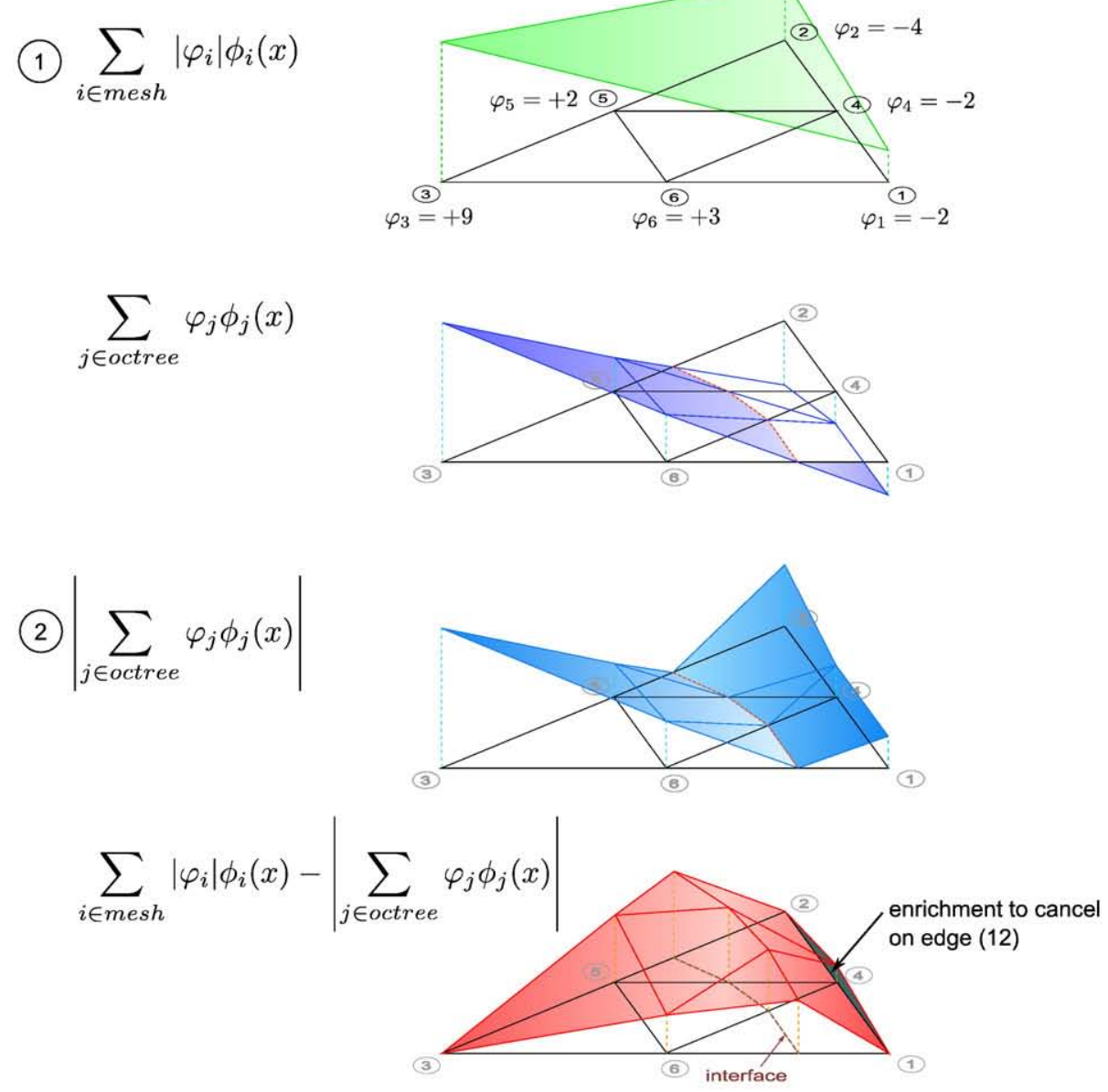

(3) Correction : Edge bubble function

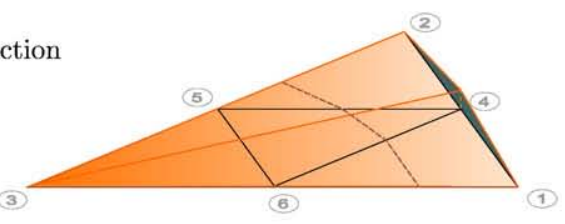

Enrichment function : $\mathbf{F}(\mathbf{x})$

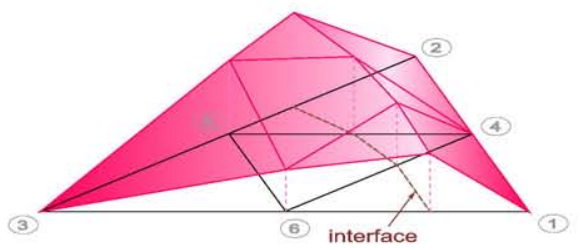

Fig. 16. Construction of the enrichment function. 
are thereafter called "non-cut edges". Fig. 16 shows that this function is no more canceled on non-cut edges, when the geometrical grid is refined.

A correction term has to be added in order to correct the enrichment function, and is called "edge bubble function" (Fig. 17).

$$
\begin{aligned}
\tilde{F}(x)= & \underbrace{\sum_{i \in \text { mesh }}\left|\varphi_{i}\right| \phi_{i}(x)}_{(1)}-\underbrace{\left|\sum_{j \in \text { octree }} \varphi_{j} \phi_{j}(x)\right|}_{(2)} \\
& -\underbrace{\left(\Pi^{\text {lin }} \varphi_{\text {Non-cut edge }}\left(\Pi^{\text {edge }} \mathrm{x}\right)-\varphi_{\text {Non-cut edge }}\left(\Pi^{\text {edge }} \mathrm{x}\right)\right)\left(\phi_{1}(\mathrm{x})+\phi_{2}(\mathrm{x})\right)}
\end{aligned}
$$

(3): edge bubble function

This enrichment Eq. (12) is computed for an element " $e$ " of the mesh (computational mesh) which is crossed by the iso-zero value of the level set. The first sum is computed on nodes $i$ of the element " $e$ ". This element is split into cells called " $e_{r}$ ", creating the octree (part of the geometrical grid). The second sum is computed on nodes $j$ of each element $e_{r}$. Shape functions of the term $\left(\phi_{1}(x)+\phi_{2}(x)\right)$ are elements shape functions. The "edge bubble function" corresponds to the level set computed on edge (12), of which the linear part is removed, and which is "stretched" to the opposite node 3.

Example. Consider a triangle element (see Fig. 18) which is crossed by the iso-zero value of the level set. We are interested in the number of non-cut edges. The iso-zero value of the level set cut two edges, so there is only one non-cut edge: edge (12). It means that an edge bubble function corresponding to edge (12) is added is in the enrichment function.

Following notations are used. Let $G$ be a Gauss point of the element, with local coordinates $(u, v)$ and barycentric coordinates $\left(\lambda_{1}, \lambda_{2}, \lambda_{3}\right)$ (Fig. 19). The projection of this point on the edge (12) gives a distance $\chi=\frac{\lambda_{2}}{\lambda_{1}+\lambda_{2}}$ from node 1 . Expressions of theses barycentric coordinates are the same than triangle shape functions $\left(\phi_{1}(x), \phi_{2}(x), \phi_{3}(x)\right)$, so barycentric coordinates are used to simplify the enrichment formulation:

$$
\begin{aligned}
\tilde{F}(x)= & \left|\varphi_{1}\right| \lambda_{1}+\left|\varphi_{2}\right| \lambda_{2}+\left|\varphi_{3}\right| \lambda_{3}-\left|\sum_{j \in \text { octree }} \varphi_{j} \lambda_{j}\right| \\
& -\left[\left|\varphi_{1}\right|\left(1-\frac{\lambda_{2}}{\lambda_{1}+\lambda_{2}}\right)+\left|\varphi_{2}\right|\left(\frac{\lambda_{2}}{\lambda_{1}+\lambda_{2}}\right)-\left|\varphi^{(12)}\left(\frac{\lambda_{2}}{\lambda_{1}+\lambda_{2}}\right)\right|\right] \\
& \times\left(\lambda_{1}+\lambda_{2}\right)
\end{aligned}
$$

$\varphi^{(12)}$ is the representation of the level set on the edge (12), with edge shape functions. The value depends on the projection of the

Gauss point on this edge $\left(\chi=\frac{\lambda_{2}}{\lambda_{1}+\lambda_{2}}\right)$ (Fig. 19).
$\quad$ Following notations are used:

- $\varphi_{i}$ : level set value on node $i$,

- $\lambda_{i}(x)=\lambda_{i}$ : barycentric coordinate of a point $x$ in the element $e$,

- $\chi$ : projection of a Gauss point on an edge (ij)

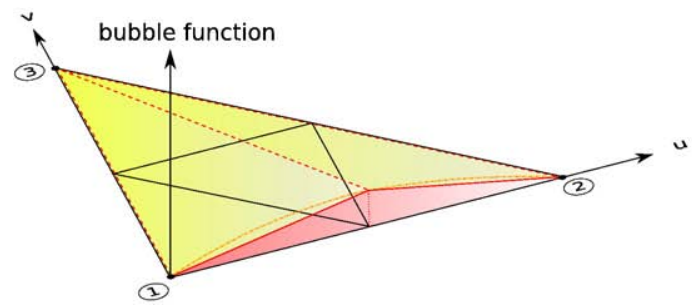

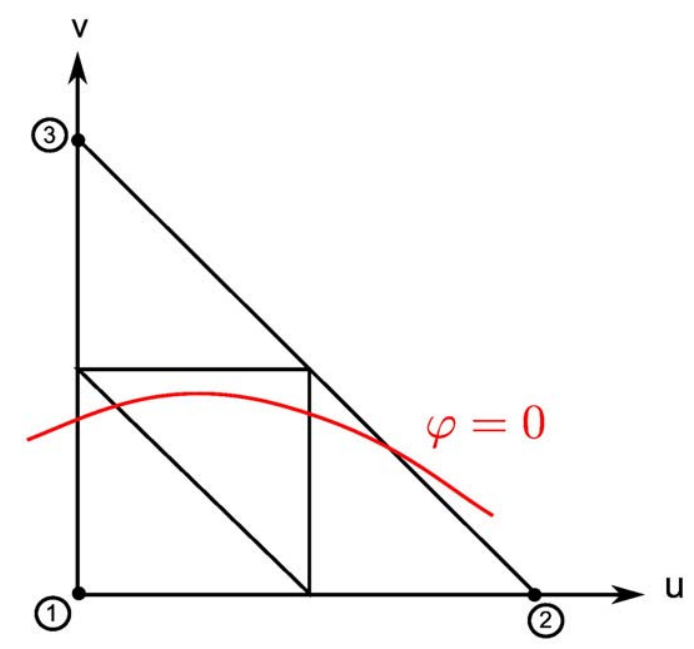

Fig. 18. Triangle element crossed by the iso-zero value of the level set.

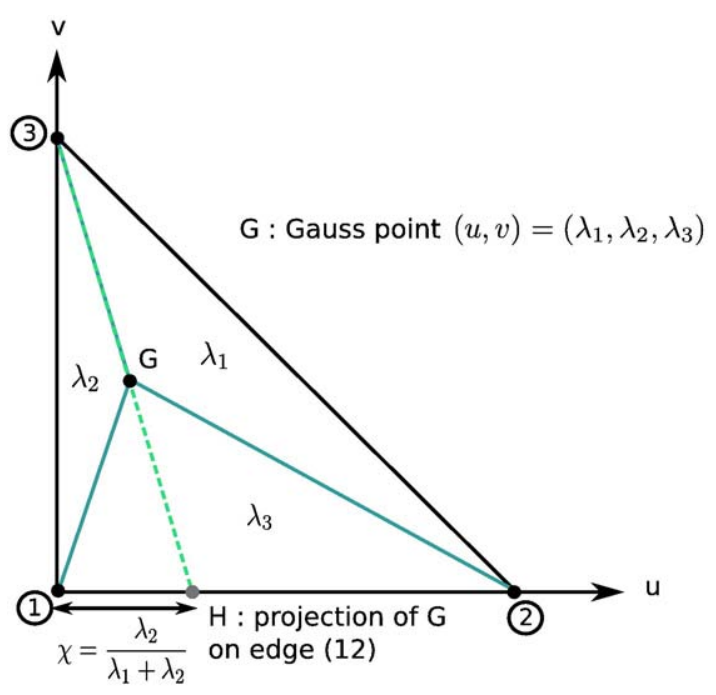

Fig. 19. Barycentric coordinates in a triangle.

- $\varphi^{(i j)}(\chi)$ : level set value on edge $(i j)$, depending on the projection $\chi$ of the Gauss point on this edge,

All level set values are computed from the geometrical grid. Finally, we obtain:

$\tilde{F}(x)=\left|\varphi_{3}\right| \lambda_{3}+\left|\varphi^{(12)}\left(\frac{\lambda_{2}}{\lambda_{1}+\lambda_{2}}\right)\right|\left(\lambda_{1}+\lambda_{2}\right)-\left|\sum_{j \in \text { octree }} \varphi_{j} \lambda_{j}\right|$

Enrichment function directly depends on edge bubble functions, and thus on the number of edges that the iso-zero value of the level set does not cut. Different configurations are presented in Fig. 20. We

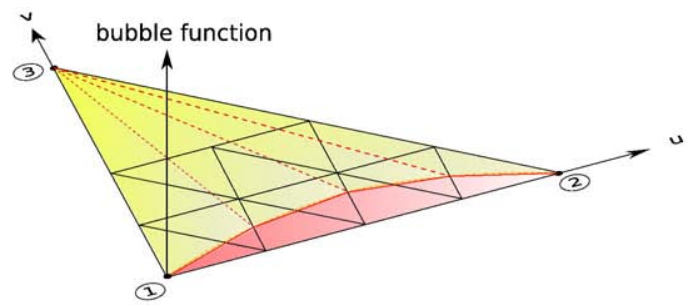

Fig. 17. Bubble function on an element: refined once and twice. 


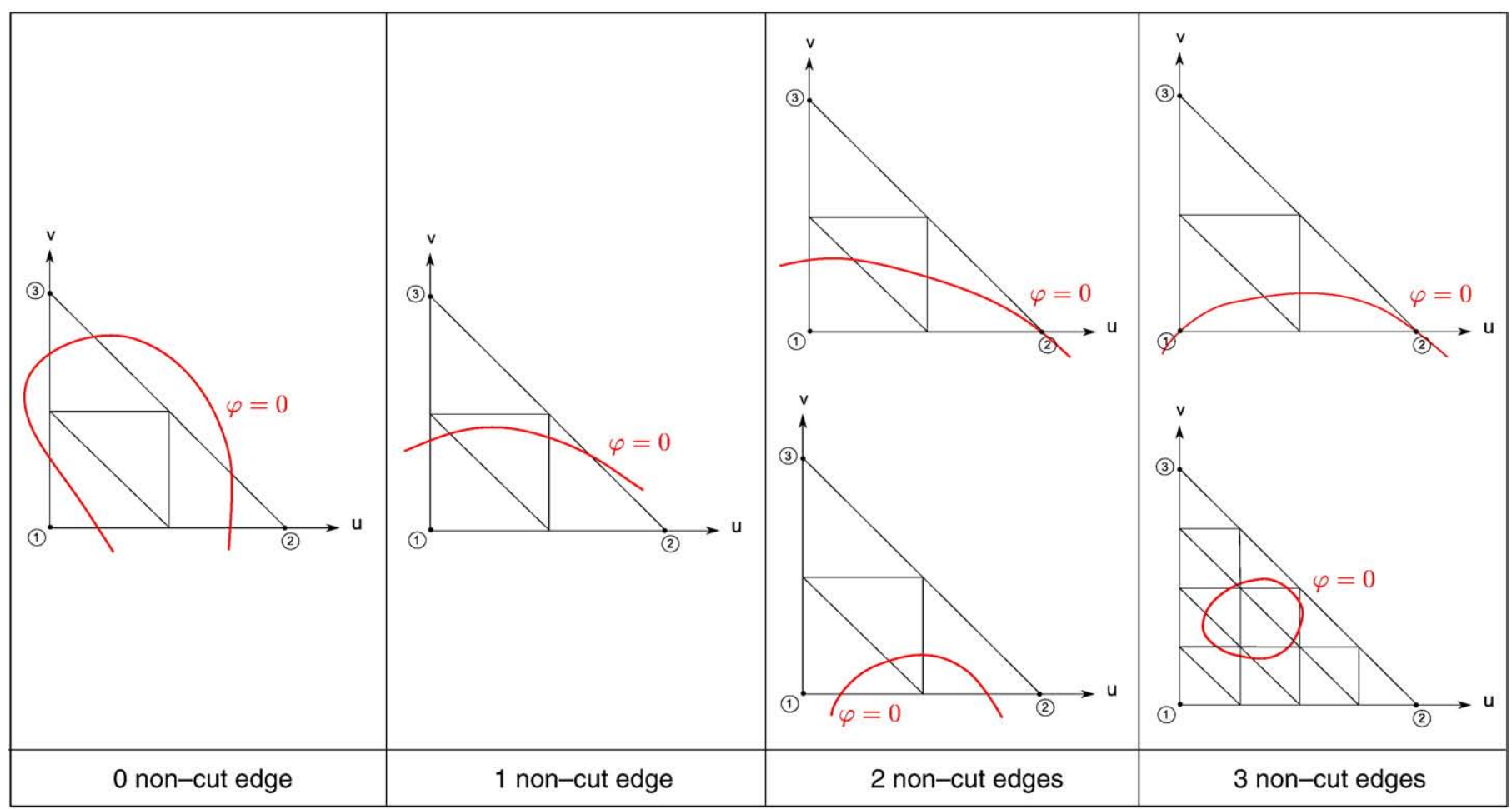

Fig. 20. Different ways for an interface to cross a triangle element.

could even imagine a discontinuity located in one element, provided that the octree is enough refined: the enrichment function is enriched by three edges bubble functions.

\subsection{Circular inclusion with imposed displacement}

The following problem is a circular inclusion $\Omega_{1}$ in the center of a material $\Omega_{2}$. A linear displacement is imposed on the boundary $\Gamma_{2}$ $(r=b)$. Material parameters are: $E_{1}=1, \nu_{1}=0.25$ (equivalent to the Lamé constants $\lambda_{1}=\mu_{1}=0,4$ ), and $E_{2}=10, \nu_{2}=0,3$ (equivalent to the Lamé constants $\lambda_{2}=5,769$ and $\mu_{2}=3,846$ ). We work with polar coordinates $(r, \theta)$.

The exact displacement solution reads:

$u_{r}(r)= \begin{cases}{\left[\left(1-\frac{b^{2}}{a^{2}}\right) \alpha+\frac{b^{2}}{a^{2}}\right] r} & \text { if } 0 \leq r \leq a \\ \left(r-\frac{b^{2}}{r}\right) \alpha+\frac{b^{2}}{r} & \text { if } a \leq r \leq b\end{cases}$

$u_{\theta}=0$

with:

$\alpha=\frac{\left(\lambda_{1}+\mu_{1}+\mu_{2}\right) b^{2}}{\left(\lambda_{2}+\mu_{2}\right) a^{2}+\left(\lambda_{1}+\mu_{1}\right)\left(b^{2}-a^{2}\right)+\mu_{2} b^{2}}$

Displacement is continuous, but with a discontinuous derivative across the interface.

And the strain solution is:

$\varepsilon_{r r}(r)=\left\{\begin{array}{cc}\left(1-\frac{b^{2}}{a^{2}}\right) \alpha+\frac{b^{2}}{a^{2}} & \text { if } 0 \leq r \leq a \\ \left.1+\frac{b^{2}}{r^{2}}\right) \alpha-\frac{b^{2}}{r^{2}} & \text { if } a \leq r \leq b\end{array}\right.$ $\varepsilon_{\theta \theta}(r)=\left\{\begin{array}{l}\left(1-\frac{b^{2}}{a^{2}}\right) \alpha+\frac{b^{2}}{a^{2}} \quad \text { if } 0 \leq r \leq a \\ \left(1-\frac{b^{2}}{r^{2}}\right) \alpha+\frac{b^{2}}{r^{2}} \quad \text { if } a \leq r \leq b\end{array}\right.$

Stresses are defined from strains:

$\sigma_{r r}(r)=2 \mu \varepsilon_{r r}+\lambda\left(\varepsilon_{r r}+\varepsilon_{\theta \theta}\right)$

$\sigma_{\theta \theta}(r)=2 \mu \varepsilon_{\theta \theta}+\lambda\left(\varepsilon_{r r}+\varepsilon_{\theta \theta}\right)$

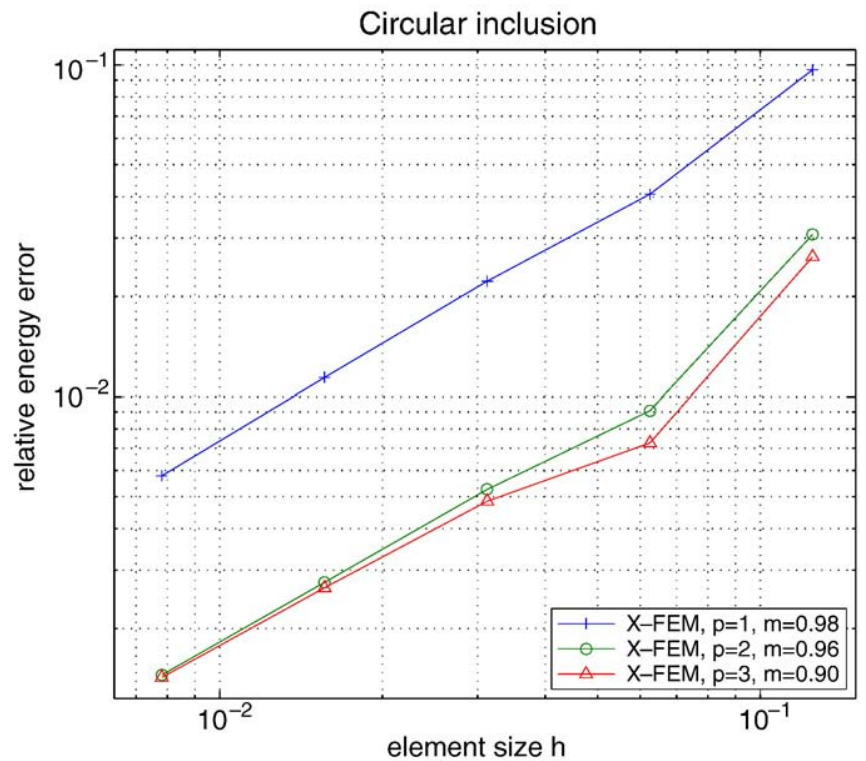

Fig. 21. Relative error norm with linear, quadratic and cubic approximation shape functions: without improving the geometrical representation. 


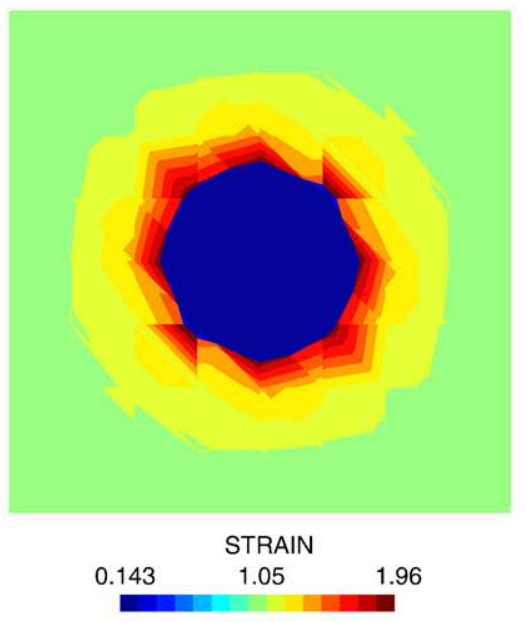

No refinement

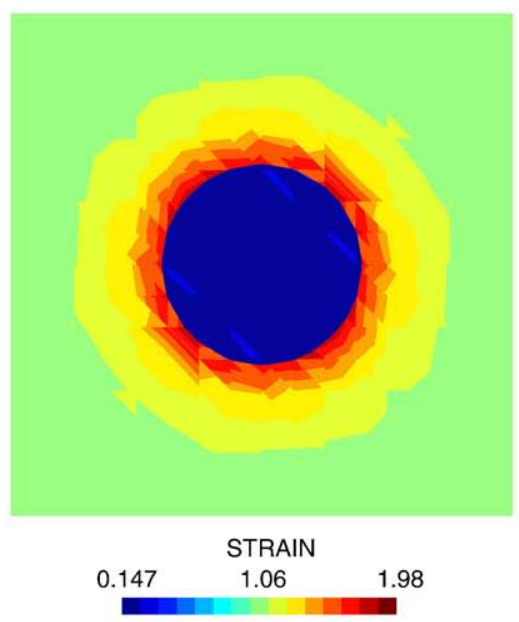

Refined once

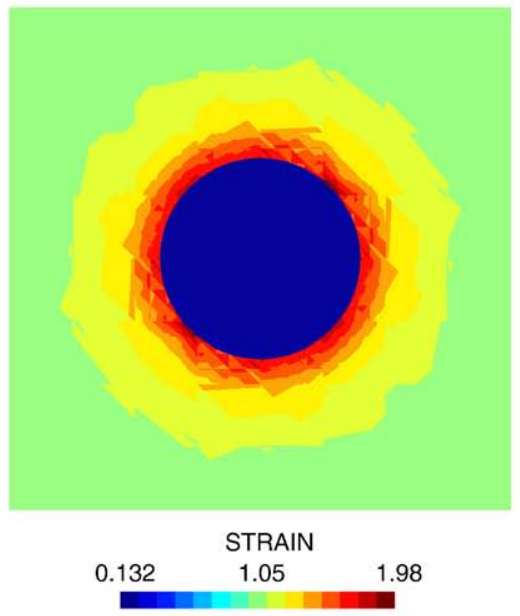

Refined twice

Fig. 22. Circular inclusion - strain with improvement of the geometry.

\section{Results}

The numerical model is a square domain $\Omega_{2}$ of side $L=2$, with a circular inclusion $\Omega_{1}$ of radius $a$ at its center. Geometrical parameters are: $b=2$ and $a=0.4$. Exact tractions are imposed on the boundary on the domain. Computations are performed with the same meshes than those for the plate with a hole.

Convergence of relative error of energy norm toward elements size, when initial enrichment function (10) is used, is presented in Fig. 21. As expected, the error decreases when quadratic and cubic approximation shape functions are used. Whereas, the precision gain is lower than the previous case of free surfaces. Optimal rates of convergence are not reached: rates of 0.93 with $p=2$, and 0.90 with $p=3$ (rates computed from energy error values of the two finest meshes).

Fig. 22 shows strains on the plate, computed using the new enrichment function (12). Strains are presented with the same coarse mesh ( 8 elements by side), but with different refinement levels of the geometrical grid: first with no refinement, then with a geometrical grid refined once and twice. Improving the geometrical representation improves the strain field around the inclusion.

As displacements are continuous, the enrichment function has also to be continuous on the domain. Therefore, the refinement of the

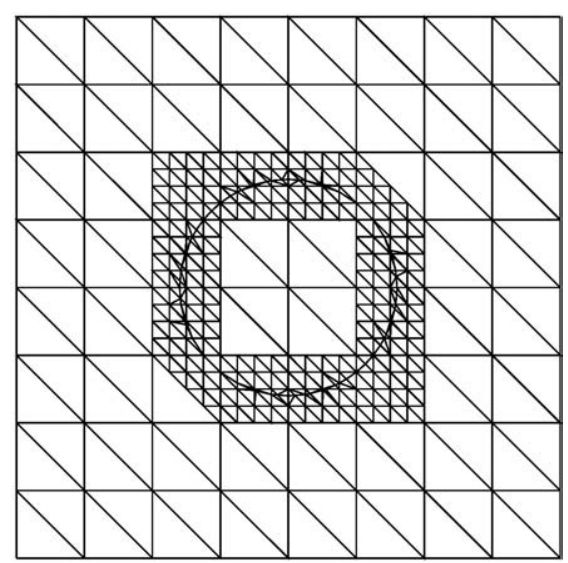

Fig. 23. Homogeneous refinement of the octree when an element is crossed by the material interface. geometrical grid is no more recursive, but homogeneous per element. Fig. 23 presents integration cells of a square mesh with 8 elements by side, created from a geometrical grid twice refined.

Results using the proposed enrichment method Eq. (12) are plotted on Figs. 24 and 25 according to elements size, for approximation orders 1,2 and 3. Initially, X-FEM converges with high order, but slower than using classical FEM, that is to say $O\left(h^{p}\right)$, where $h$ is the element size and $p$ the polynomial basis order. Improving the geometrical representation reduces energy error. However, contrary to the previous application (plate with a hole), optimal rates of convergence cannot be reached with an optimal depth of refinement. Indeed, the required refinement level of the geometrical grid is more important with a material interface; but for a given mesh, energy error reaches a limit when the refinement level increases.

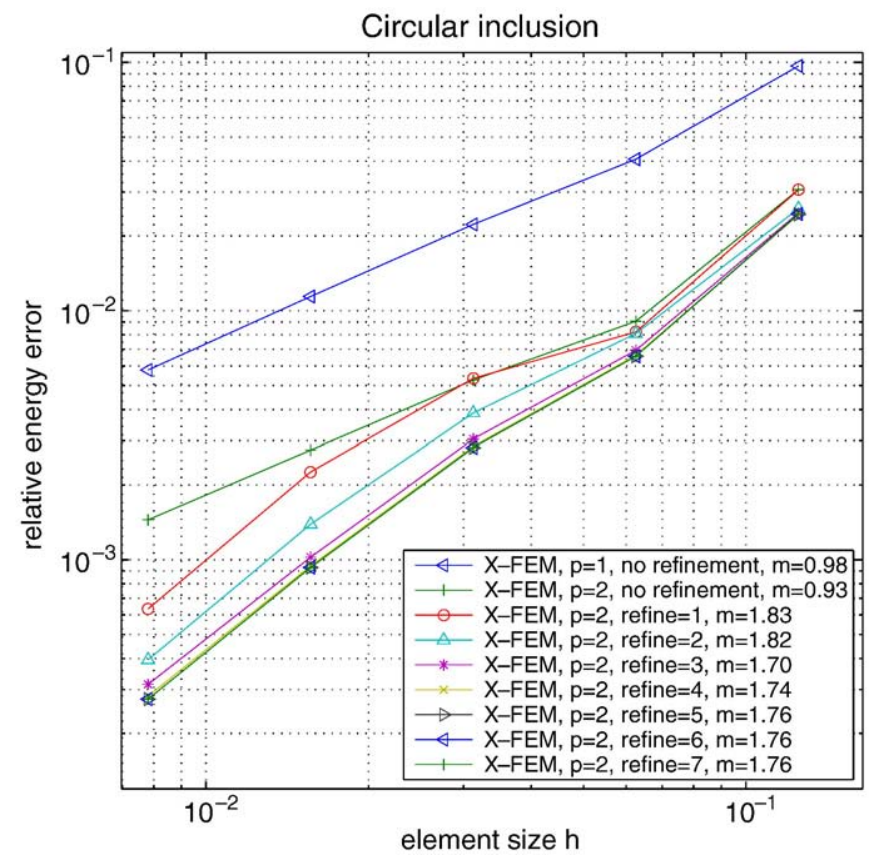

Fig. 24. Relative error norm with quadratic approximation shape functions with the proper enrichment function. 


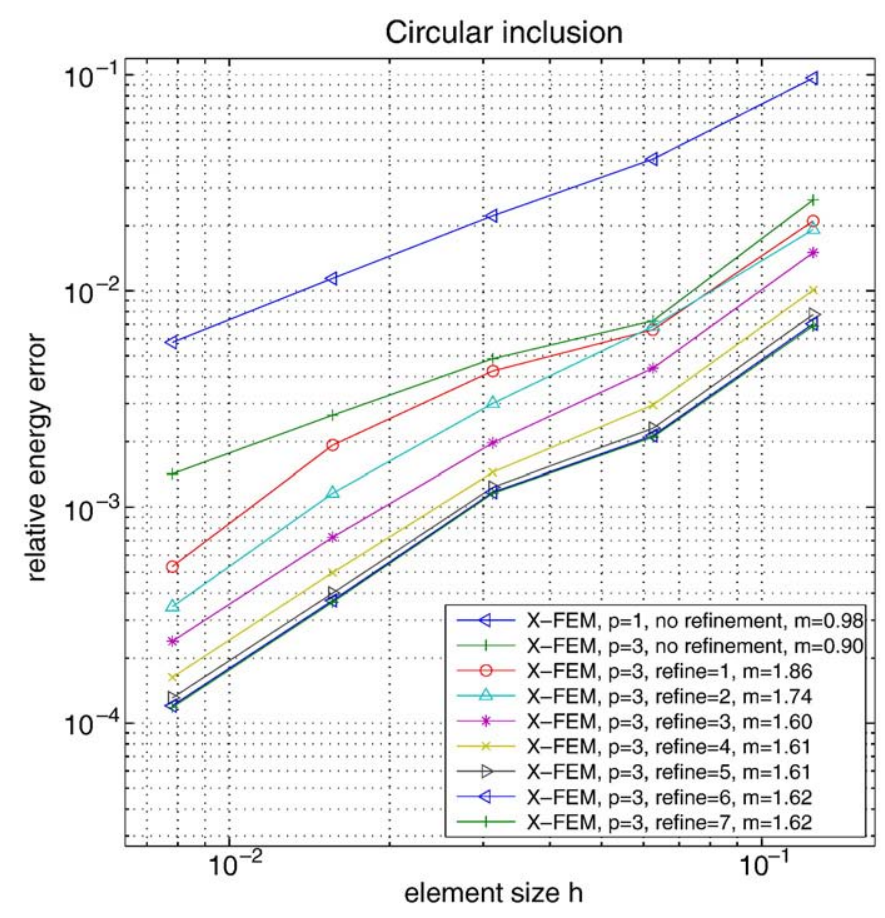

Fig. 25. Relative error norm with cubic approximation shape functions with the proper enrichment function.

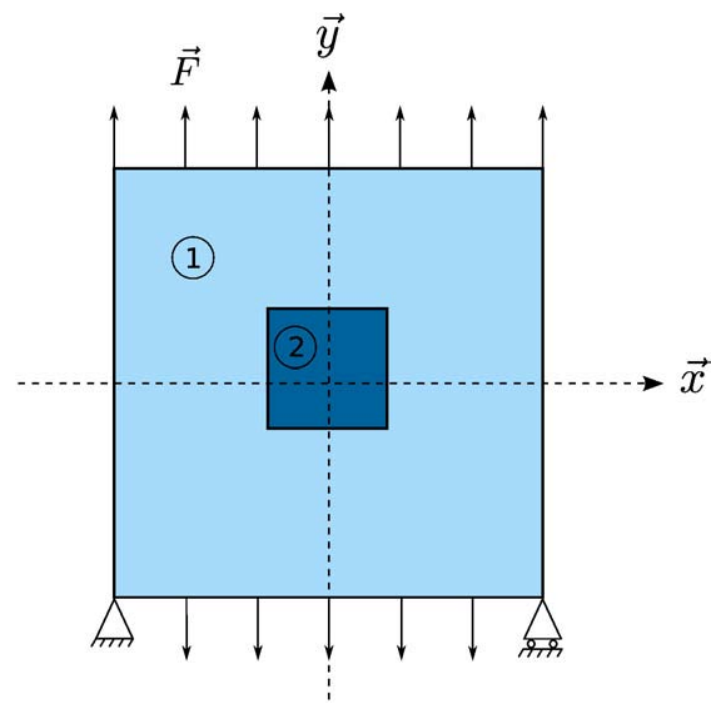

Fig. 26. Plate with a squared inclusion under uniaxial tension.

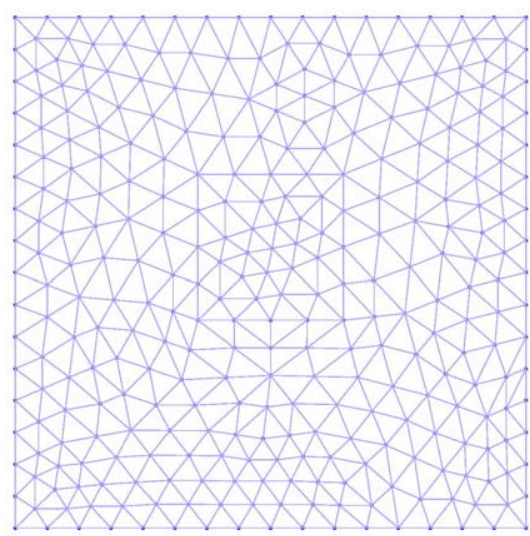

Nevertheless, the improvement of the geometrical representation and the proposed X-FEM enrichment enable the use of higher order approximation shape functions, reducing considerably energy error.

\subsection{Material interface with geometric singularities}

Up to now, only smooth interfaces were considered. The proposed enrichment function can also be applied to inclusion with geometric singularities as polygonal shape. In order to compare results from classical FEM and X-FEM, a plate with a squared inclusion under uniaxial tension is studied.

The numerical model is a square domain of length $L=2$, with a square inclusion of side $L=0.57$ and of center $(0,0.1)$ (Fig. 26). The finite element solution is computed on a fine mesh with 400 triangular elements by side. Its element edges respect the material interface, and particularly the four corners. For example, a finite element mesh with 16 elements by side is shown in Fig. 27. The finite element solution is the reference solution. X-FEM meshes are square regular grid with 8, 16 (Fig. 27), 32, 64, and 128 triangular elements by side. All meshes are generated with GMSH. Corners of the material interface are contained in elements of X-FEM meshes, in order to study the influence of the enrichment function.

Fig. 28 shows enrichment function computed on a regular mesh with 16 elements by side, with a geometrical grid twice refined. Using the initial enrichment function [21], corners of the interface are truncated if elements do not respect this interface. By contrast, refining the geometrical grid allows the enrichment function to introduce a discontinuous derivative closer to the real position of the material interface.

$\mathrm{X}$-FEM solutions are compared to the finite element reference solution. Energy error norms with linear, quadratic and cubic shape functions are shown in Figs. 29-31. This enrichment only introduce a discontinuous derivative in the displacement field. Geometrical singularities as corners of the squared interface lead to singularities in the field. The material interface enrichment cannot represent these singularities. This is why refinement only can reduce energy error norm, and is not able to improve convergence rates.

To obtain optimal rates of convergence, singular functions should be added to the finite element approximation, to be able to represent the behavior of the squared material interface. For example, to represent a crack propagation, the displacement field is enriched by a discontinuous field along a crack and by asymptotic fields near crack tips [22].

\section{Conclusion}

This article studies the possibility to use higher order approximation shape functions with X-FEM, when geometries include curved discontinuities. In particular, we are interested by curved free surfaces

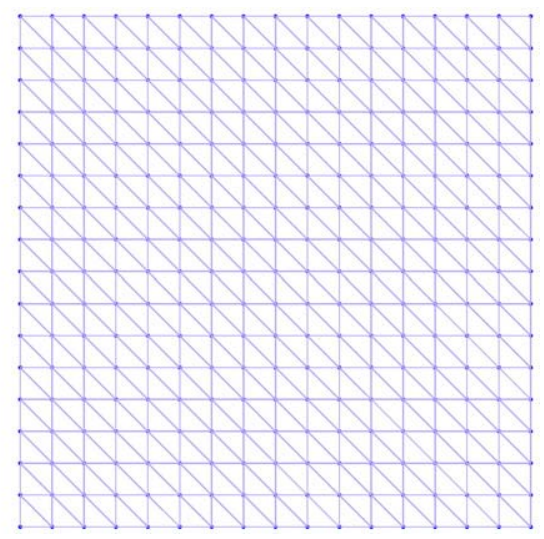

Fig. 27. FEM mesh with 16 elements by side (left), X-FEM mesh with 16 elements by side (right). 


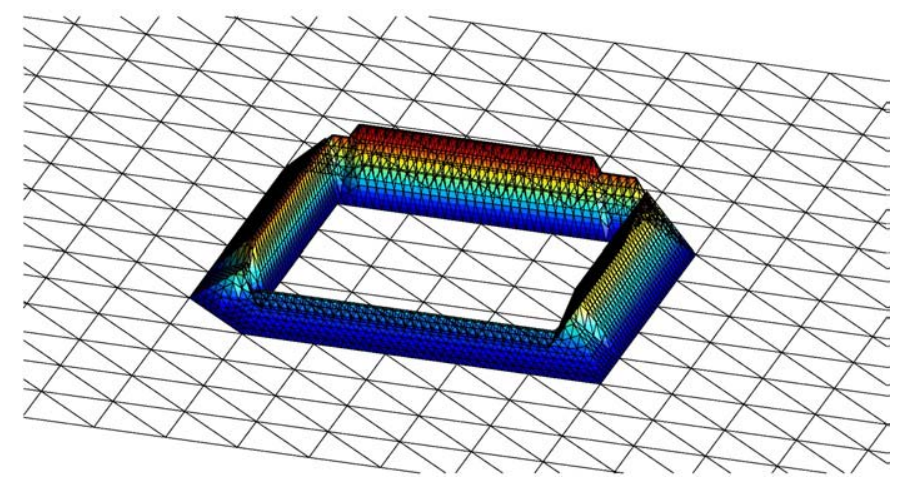

Fig. 28. Enrichment function for a squared material interface.

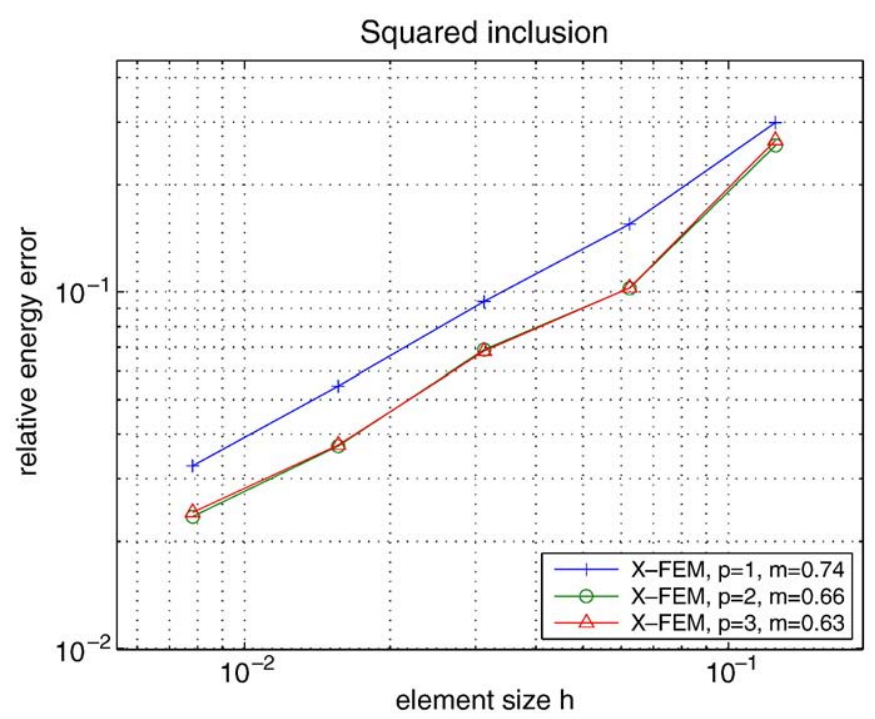

Fig. 29. Squared inclusion: energy error norm with linear approximation shape functions.

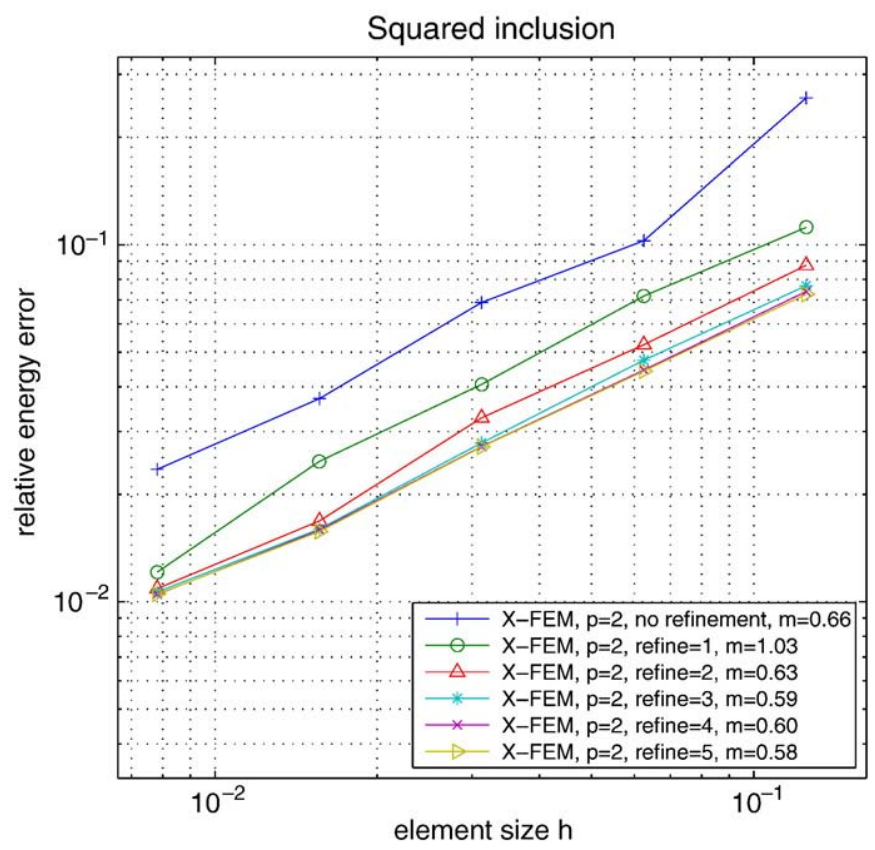

Fig. 30. Squared inclusion: energy error norm with quadratic approximation shape functions with the material interface enrichment function.

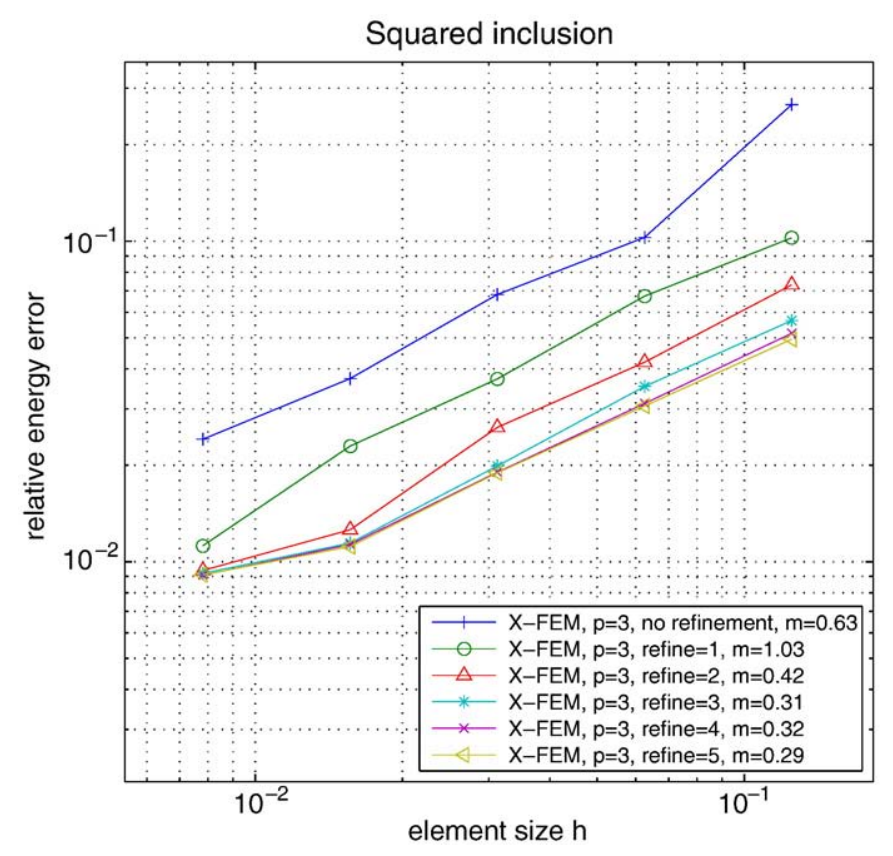

Fig. 31. Squared inclusion: energy error norm with cubic approximation shape functions with the material interface enrichment function.

and material interfaces. A geometrical improvement is done refining the level set representation. Moreover, a new enrichment function is required in the case of material interfaces. Convergences studies are done with two numerical examples in two dimensions: a plate with a hole under uniaxial tension, and a circular inclusion with imposed displacement. Results show that improving the representation of geometry allows to reduce energy error. Considering the plate with a hole, rates of convergence are close to classical FEM ones, that is to say $O\left(h^{p}\right)$, with $\mathrm{h}$ the element size, and $\mathrm{p}$ the order of approximation shape function. This approach gets rid of difficulties met during mesh generation, as mesh do not have to respect discontinuities with XFEM. Operations to represent geometries are simplified, and complex geometries can be represented on coarse meshes.

Study of curved crack representation will be presented in a forthcoming paper, along with development of the proposed approach in three dimensions.

\section{References}

[1] T. Belytschko, T. Black, Elastic crack growth in finite elements with minimal remeshing, Int. J. Numer. Methods Engrg. 45 (1999) 601-620.

[2] K. W. Cheng, T.-P. Fries. Higher-order xfem for curved strong and weak discontinuities. Int. J. Numer. Methods Engrg. doi:10.1002/nme.2768.

[3] C. Daux, N. Moës, J. Dolbow, N. Sukumar, T. Belytschko, Arbitrary branched and intersecting cracks with the extended finite element method, Int. J. Numer. Methods Engrg. 48 (2000) 1741-1760.

[4] S. Dey, R.M. O'Bara, M.S. Shephard, Curvilinear mesh generation in 3d, Computer Aided Geometry Design 33 (2001) 199-209.

[5] C.A. Duarte, J.T. Oden, An h-p adaptive method using clouds, Comput. Meth. Appl. Mech. Engrg. 139 (1996) 237-262.

[6] A. Düster, J. Parvizian, Z. Yang, E. Rank, The finite cell method for threedimensional problems of solid mechanics, Comput. Meth. Appl. Mech. Engrg. 197 (2008) 3768-3782.

[7] T.-P. Fries, A corrected xfem approximation without problems in blending elements, Int. J. Numer. Methods Engrg. 75 (2008) 503-532.

[8] T.-P. Fries, H.-G. Matthies, Classification and Overview of Meshfree Methods, Institute of Scientific Computing, Technical University Braunschweig, Brunswick, Germany, 2003.

[9] P.-L. George, Mesh Generation, Application To Finite Elements, Hermes Science Publication, 2000.

[10] C. Geuzaine, J.-F. Remacle, Gmsh: A Three-Dimensional Finite Element Mesh Generator with Built-in Pre- and Post-Processing facilities, 2009.

[11] T. Hughes, J. Cottrell, Y. Bazilevs, Isogeometric analysis: CAD, finite elements, nurbs, exact geometry and mesh refinement, Comput. Meth. Appl. Mech. Engrg. 194 (2005) 4135-4195. 
[12] T. Hughes, A. Reali, G. Sangalli, Efficient quadrature for nurbs-based isogeometric analysis. Computational Geometry and Analysis 199 (5-8), 301-313.

[13] E. Iarve, Mesh independent modelling of cracks by using higher order shape functions, Int. J. Numer. Methods Engrg. 56 (2003) 869-882.

[14] G. Királyfalvi, B.A. Szabó, Quasi-regional mapping for the p-version of the finite element method, Finite Elem. Anal. Des. 27 (1997) 85-97.

[15] P. Laborde, J. Pommier, Y. Renard, M. Salan, High-order extended finite element method for cracked domains, Int. J. Numer. Methods Engrg. 64 (2005) 354-381.

[16] A. Legay, H.W. Wang, T. Belytschko, Strong and weak arbitrary discontinuities in spectral finite elements, Int. J. Numer. Methods Engrg. 64 (2005) 991-1008.

[17] Xiao-Juan Luo, Mark Shephard, Robert O'Bara, Rocco Nastasia, Mark Beall, Automatic p-version mesh generation for curved domains, Engrg. Comput. 20 (2004) 273-285.

[18] X.J. Luo, M.S. Shephard, J.-F. Remacle, R.M. O'Bara, M.W. Beall, B.A. Szabó, R. Actis, p-version mesh generation issues, th International Meshing Roundtable, Sandia National Laboratories, 2002, pp. 343-354.

[19] E. Marchandise, P. Geuzaine, N. Chevaugeon, J.-F. Remacle, A stabilized finite element method using a discontinuous level set approach for the computation of bubble dynamics, J. Comput. Phys. 225 (2007) 949-974.

[20] J. Melenk, I. Babuska, The partition of unity finite element method: basic theory and applications, Comput. Meth. Appl. Mech. Engrg. 139 (1996) 289-314.

[21] N. Moës, M. Cloirec, P. Cartraud, J.-F. Remacle, A computational approach to handle complex microstructure geometries, Comput. Meth. Appl. Mech. Engrg. 192 (2003) 3163-3177.

[22] N. Moës, J. Dolbow, T. Belytschko, A finite element method for crack growth without remeshing, Int. J. Numer. Methods Engrg. 46 (1999) 131-150.
[23] S. Osher, R. Fedkiw, Level Set Methods and Dynamic Implicit Surfaces, Applied Mathematical Sciences, 153, Springer, 2003.

[24] J.P. Pereira, C.A. Duarte, D. Guoy, X. Jiao, hp-generalized fem and crack surface representation for non-planar 3-d cracks, Int. J. Numer. Methods Engrg. 77 (2008) 601-633.

[25] M.S. Shepard, J.E. Flaherty, K.E. Jansen, X. Li, X. Luo, N. Chevaugeon, J.-F.R., Mark W. Beall, R.M. O'Bara, Adaptive mesh generation for curved domains, Appl. Numer. Math. 52 (2005) 251-271.

[26] S.J. Sherwin, J. Peiró, Mesh generation in curvilinear domains using high-order elements, Int. J. Numer. Methods Engrg. 53 (2002) 207-223.

[27] F.L. Stazi, E. Budyn, J. Chessa, T. Belytschko, An extended finite element method with higher-order elements for curved cracks, Comput. Mech. 31 (2003) 38-48.

[28] N. Sukumar, D.L. Chopp, N. Moës, T. Belytschko, Modeling holes and inclusions by level sets in the extended finite-element method, Comput. Meth. Appl. Mech. Engrg. 190 (2001) 6183-6200.

[29] N. Sukumar, N. Moës, T. Belytschko, B. Moran, Extended finite element method for three-dimensional crack modelling, Int. J. Numer. Methods Engrg. 48 (2000) 1549-1570.

[30] B. Szabó, I. Babuska, Finite Element Analysis, Wiley, 1991.

[31] J.F. Unger, C. Könke, Simulation of concrete using the extended finite element method, Proceedings of International Conference on Computational Modelling of Concrete Structures, 2006, pp. 239-247.

[32] G. Ventura, On the elimination of quadrature subcells for discontinuous functions in the extended finite element method, Int. J. Numer. Methods Engrg. 66 (2006) 761-795.

[33] O.C. Zienkiewicz, R.L. Taylor, The Finite Element Method, Fifth edition, The Basis, 1, Butterworth-Heinemann, 2000. 